\title{
Bubble dynamics in time-periodic straining flows
}

\author{
By I. S. KANG $†$ AND L. G. LEAL $\ddagger$ \\ Department of Chemical Engineering, California Institute of Technology, \\ Pasadena, CA 91125, USA
}

(Received 16 March 1989 and in revised form 17 January 1990)

The dynamics and breakup of a bubble in an axisymmetric, time-periodic straining flow has been investigated via analysis of an approximate dynamic model and also by time-dependent numerical solutions of the full fluid mechanics problem. The analyses reveal that in the neighbourhood of a stable steady solution, an $O\left(\epsilon^{\frac{1}{2}}\right)$ timedependent change of bubble shape can be obtained from an $O(\epsilon)$ resonant forcing. Furthermore, the probability of bubble breakup at subcritical Weber numbers can be maximized by choosing an optimal forcing frequency for a fixed forcing amplitude.

\section{Introduction}

One of the outstanding unsolved problems in multiphase flow theory is the conditions for bubble breakup at high Reynolds number. Most existing literature suggests the existence of a critical Weber number, based upon dimensional analysis, that is invariant to the details of the flow. However, recent theoretical studies of bubble deformation in steady uniaxial and biaxial straining flow have shown that the concept of a single, universal critical Weber number is inadequate. At the very least, the critical Weber number depends strongly on the flow type, and on the Reynolds number. Furthermore, for simple step changes in the magnitude of the velocity gradient, theoretical studies also demonstrated a sensitivity of the breakup criteria to the initial bubble shape (and, thereby, by inference, to the flow history).

In the present paper, we continue our investigation of bubble deformation and breakup at high Reynolds number, by considering the dynamic response of the bubble shape to time-periodic perturbations of a uniaxial straining flow. The steadyflow version of this problem has previously been studied by many investigators, including Miksis (1981), Ryskin \& Leal (1984) and Kang \& Leal (1987, 1988). As a result, the dynamics of changes in bubble shape starting from a variety of initial conditions, as well as the properties of steady-state solutions, including instabilities and limit points, are all fairly well understood. This work is reviewed briefly in $\S 3$. Of particular relevance to the present study is the phase-plane portrait of changes of bubble shape, which demonstrates the existence of a homoclinic orbit (or separatrix) in the inviscid limit, which separates stable oscillatory solutions from unstable solutions that correspond to exponential stretching in the principal strain direction. This suggests that the bubble shape will be susceptible to a transition from regular to chaotic behaviour upon introduction of a time-dependent modulation of the uniaxial straining flow.

+ Present address: Chemical Engineering Department, POSTECH, P.O. Box 125, Pohang, 790 Korea.

$\ddagger$ Present address: Dept. of Chemical and Nuclear Engineering, UCSB, Santa Barbara, CA 93106, USA. 


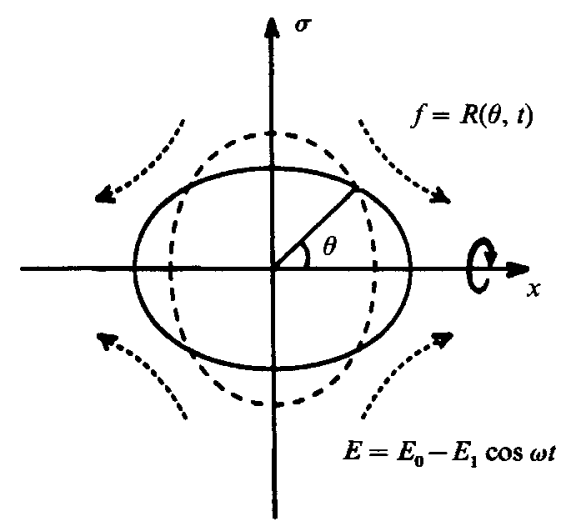

Figure 1. A bubble in a time-periodic uniaxial straining flow.

In the present paper, we consider a single-frequency, time-periodic straining flow. This problem may be viewed as a first qualitative model for investigation of bubble dynamics in more complicated flows such as the motion through a wavy channel, or in a stirred tank, or near the propellor of a ship, and so on. As we shall see, the analysis reveals the existence of a resonant amplification in the magnitude of shape oscillations at a critical forcing frequency, as well as the possibility of bubble breakup via chaotic oscillations of shape at subcritical Weber numbers.

The main part of this paper consists of the analysis of a simple low-dimensional dynamical model for changes in bubble shape that has been devised on the basis of known results for the steady straining flow problems. However, at the end of the paper, we also present exact time-dependent numerical solutions for the full fluid mechanics problem to corroborate the conclusions from the model analysis. It is also noted that the dynamical model is directly applicable to time-dependent oscillations in the radius of a spherical bubble in a time-periodic pressure field, and to the dynamics of changes in the shape of an inviscid drop subjected to small periodic perturbations of a uniform electric field.

\section{Problem statement}

We consider an incompressible gas bubble of volume $\frac{4}{3} \pi a^{3}$, which is undergoing deformations of shape in the presence of a time-periodic, axisymmetric uniaxial extensional flow of a fluid with density $\rho$ and viscosity $\mu$ as sketched in figure 1 . The surface of the bubble is described by the shape function, $r=f=R(\theta, t)$, and is characterized completely by a uniform surface tension $\gamma$. The undisturbed flow far from the bubble is given by

$$
\boldsymbol{u}^{\prime}=E \cdot \boldsymbol{r}^{\prime}, \quad \boldsymbol{E}=\boldsymbol{E}(t)\left(\begin{array}{ccc}
1 & 0 & 0 \\
0 & -\frac{1}{2} & 0 \\
0 & 0 & -\frac{1}{2}
\end{array}\right), \quad E>0,
$$

where $E(t)$ is the time-periodic principal strain rate (e.g. $\left.E(t)=E_{0}-E_{1} \cos \omega t\right)$. Important parameters for this problem include, in addition to $\omega$ and $E_{1} / E_{0}$, the dimensionless numbers

$$
W_{0}=\frac{2 \rho\left(E_{0} a\right)^{2} a}{\gamma}, \quad S=\frac{\left(\rho a^{3} / \gamma\right)^{\frac{1}{2}}}{\rho a^{2} / \mu},
$$

where $W_{0}$ is the Weber number for the case of constant strain rate and $S$ is the ratio of the surface-tension-based timescale and the viscous-diffusion timescale. 
(a)
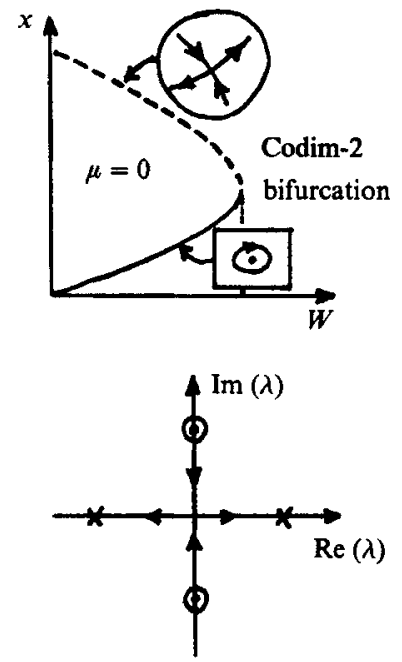

(b)
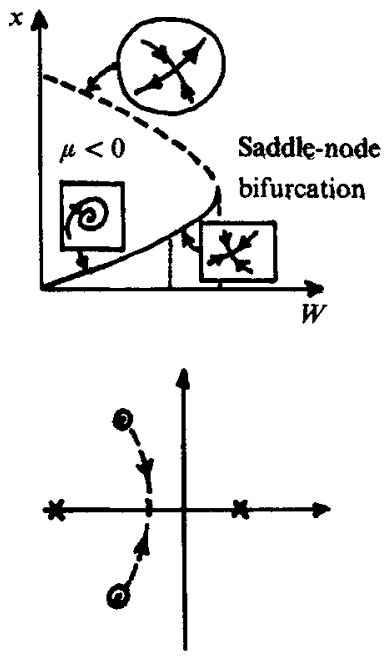

$x=\left\langle R, P_{3}\right\rangle$

FIGURe 2. A schematic representation of known results for bubble dynamics in a steady, uniaxial straining flow; the existence of steady solutions, phase portraits, and eigenvalues from linear stability analysis.

\section{Review of bubble dynamics in steady straining flows}

Before going on to the main subject, let us start with a discussion of the bubble dynamics in steady straining flows $(E=0)$, which is the base flow problem for the time-periodic straining flows that are considered in the remainder of the paper. For discussion in this and the following sections, we adopt a scalar measure of deformation, $x$, which is defined as

$$
x=\left\langle R(\theta, t), P_{2}(\cos \theta)\right\rangle=\int_{0}^{\pi} R(\theta, t) P_{2}(\cos \theta) \sin \theta \mathrm{d} \theta .
$$

The known results for the steady flow problem are summarized in figure 2, where we plot $x$ versus Weber number for both $\mu=0$ and $\mu>0$. The first contribution to this problem was made by Miksis (1981), who used the boundary-integral technique in conjunction with Newton's method to predict steady-state shapes for the potential flow limit. As shown in figure 2(a), Miksis showed that there exist multiple steady solutions for a certain Weber-number range, and no steady solutions for $W$ beyond a maximum critical value, $W_{c}$. Later Ryskin \& Leal (1984) obtained the stable branch (emanating from the spherical steady state) for a number of non-zero-viscosity cases by using a finite-difference technique coupled with boundary-fitted orthogonal mapping.

Analytical results were first obtained in an earlier paper of this series, Kang \& Leal (1988), using the domain perturbation technique. The most important results were the stability characteristics also shown in figure 2 , based upon the assumption that the steady shape is approximately spherical. In particular, for $\mu=0$, the eigenvalues, corresponding to perturbations about the lower ('stable') steady solution branch, were shown to be strictly imaginary and decreasing in magnitude as $W$ is increased until they become zero at a certain Weber number (shown by full unsteady numerical solutions - Kang \& Leal (1987) - to correspond precisely to the limit point, $W_{\mathrm{c}}$, 
predicted by Miksis (1981)). Beyond this (i.e. for larger $x$ ) on the upper solution branch, the eigenvalues for $\mu=0$ are real (one positive and one negative), and the steady-state solutions are unstable. For $\mu>0$, on the other hand, the eigenvalues for small $W$ on the lower solution branch are complex with negative real parts, indicating stability via a decaying oscillatory solution mode. Then for larger $W$ on the same branch, the eigenvalues are both real and negative, again indicating stability but via a monotonic decay, until finally at $W=W_{\mathrm{c}}$, one eigenvalue becomes zero and then increasingly positive for larger $x$, thus indicating again that the upper solution branch is unstable. As we shall see later, the fact that the frequency for $\mu=0$ decreases and becomes zero at the limit point implies extremely important consequences for the bubble dynamics. Since the two eigenvalues for the disturbance equation near the steady state become zero simultaneously, we have a so-called codimension- 2 bifurcation point, and this means that all of the possible, qualitative dynamical features near the critical point in parameter space can be deduced via the universal unfolding theory (e.g. Guckenheimer \& Holmes 1983).

\section{Model equation for the dynamics of changes in bubble shape in time- periodic straining flows}

As already stated in the introduction, the main topic of the present paper is the dynamic response of a deformable bubble to a time-periodic, axisymmetric straining flow. The most desirable analytic approach is, of course, a rigorous solution of the full problem, but that is not a practical alternative. On the other hand, one can solve the exact problem numerically over a considerable range of the parameter space, and this is a practical goal, especially in view of the axisymmetry of the problem. The limitation of a purely numerical approach is the necessity of solving a very large number of special cases to obtain a general understanding (note that the problem is characterized by four parameters, $W_{0}, S$, and the amplitude and frequency of the periodic contribution to the strain rate, as well as the initial bubble shape and rate of change of shape). Thus, we first consider a dynamical model to explore the qualitative characteristics of the bubble response to a time-periodic strain rate. Although approximate ( $a d h o c)$, this model is consistent with known results for smallamplitude shape oscillations in a quiescent fluid, due to Lamb (1932), and for deformation in a steady straining flow, as described in the previous section. Furthermore, it is consistent with the expected dynamical model derived via the universal unfolding theory for the local behaviour near a critical point at which both eigenvalues vanish (i.e. a double-zero-eigenvalue critical point). Thus, it is guaranteed to exhibit the correct (qualitative) dynamical features, at least in the vicinity of the critical point. Nevertheless, the model is approximate and ad hoc, and we therefore also obtain a limited number of exact numerical solutions of the full Navier-Stokes equations, in order to corroborate the model predictions.

We begin by seeking a low-dimensional model equation to describe the dynamics of bubble deformation in a time-dependent straining flow. For this purpose, we use the scalar measure of deformation $x$, as defined in (2), which is known from previous studies to provide a qualitatively correct description of deformation in steady axisymmetric straining flows. The variable $x$ is a measure of the magnitude of the $P_{2}$ mode of deformation, which is likely to provide a useful measure of deformation only when the deformed shape is axisymmetric (or nearly axisymmetric). Obviously, $x$ is zero when the bubble is spherical. Increasing values of $x$ can be viewed as a measure 
of increasing bubble elongation along the symmetry axis, relative to the case of a sphere.

As discussed in $\S 3$, there are several analytical results for the corresponding steady flow problem which should be reflected in the model, dynamical equation. In particular, for the case of $|x|,|\dot{x}| \ll 1$, and $W=0$, Lamb (1932) derived the following equation to describe shape oscillations in a quiescent, viscous fluid:

$$
\ddot{x}+(40 S) \dot{x}+12 x=0,
$$

where $S$ is a dimensionless number that is proportional to the viscosity (see the definition below (1)). Recently, Kang \& Leal (1988) extended this result to non-zero Weber numbers using a direct integration of the normal stress balance over the bubble surface. The steady-state small-deformation solution, for the case of constant Weber number $W(W \geqslant 0)$, was also obtained by Kang \& Leal (1988) in the form

$$
67 x-755 x^{2}+O\left(x^{3}\right)=W
$$

and this accounts for the existance of a limit point at a critical value of $W$, and two steady solutions for smaller $W$.

Finally, we include the time-dependency of the strain rate, as given by (1), by assuming that the Weber number is a periodic function of time in the form,

$$
W(t)=W_{0}-W_{1} \cos \omega t
$$

Then, to achieve a dynamical model in the form

$$
\ddot{x}=f(x, \dot{x}, S, W(t))
$$

that reflects all of the qualitative features of $(3)-(5)$, we combine these equations in the ad hoc form

$$
\ddot{x}=K W_{0}-\left(a x-b x^{2}\right)-\mu^{\prime} \dot{x}-\delta^{\prime} \cos \omega t,
$$

where

$$
K=\frac{12}{67}, \quad a=12, \quad b=\frac{12 \times 755}{67}, \quad \mu^{\prime}=40 S, \quad \delta^{\prime}=K W_{1} .
$$

The above equation can be simplified further by rescaling the variables as

$$
\tilde{t}=\left(\frac{a}{2}\right)^{\frac{1}{2}} t, \quad \tilde{x}=x \frac{2 b}{a}, \quad \tilde{w}=\frac{4 b K}{W_{0} a^{2}}, \quad \epsilon \tilde{\mu}=\left(\frac{2}{a}\right)^{\frac{1}{2}} \mu^{\prime}, \quad \epsilon \tilde{\delta}=\delta^{\prime} \frac{4 b}{a^{2}}, \quad \tilde{\omega}=\left(\frac{2}{a}\right)^{\frac{1}{2}} \omega,
$$

where we have introduced a small parameter $\epsilon$ to take account of the effects of smallamplitude time-periodic forcing and small viscosity, in which we have our primary interests. On dropping tildes, for simplicity, the model equation in rescaled form is

$$
\ddot{x}=w-\left(2 x-x^{2}\right)-\epsilon(\mu \dot{x}+\delta \cos \omega t) .
$$

Now it is quite easy to show that the model equation (7) has the correct qualitative features at least for the cases of constant Weber number $(\epsilon \delta=0)$. First, we can see that it has two steady-state solutions for $w<1$, but no solution for $w>1$. For $w<$ 1 , the two steady solutions are $x_{\mathrm{s}}=1 \pm(1-w)^{\frac{1}{2}}$. Secondly, the steady solutions have the same stability characteristics as shown in figure 2 . In particular, the eigenvalues for small disturbances near the steady-state solution $x_{\mathrm{s}}$ are

$$
\lambda_{ \pm}=\frac{1}{2}\left\{-(\epsilon \mu) \pm\left[(\epsilon \mu)^{2}-8\left(1-x_{\mathrm{s}}\right)\right]^{\frac{1}{2}}\right\} .
$$

Therefore, if $\mu=0$, we have two pure imaginary eigenvalues for $x_{\mathrm{s}}<1$, and two real 
eigenvalues with different signs for $x_{s}>1$. If $\mu>0$, we have two complex eigenvalues with negative real parts for $0 \leqslant x_{\mathrm{s}}<1-\frac{1}{8}(\epsilon \mu)^{2}$, two negative real eigenvalues for $1-\frac{1}{8}(\epsilon \mu)^{2}<x_{\mathrm{s}}<1$, and one negative and one positive real eigenvalue for $x_{\mathrm{s}}>1$. Hereafter, we denote the two steady-state solutions by $x_{\mathrm{us}}$ and $x_{\mathrm{ss}}$ depending on their stability.

\section{Unfolding of the double-zero-eigenvalue critical point}

It is evident, from the above discussion, that the simple dynamical model, given by (7), exhibits all of the known qualitative features for small-amplitude bubble deformation in a steady straining flow. However, the more important question for present purposes is whether it faithfully reproduces the dynamic response of the bubble in a time-dependent flow. One way to answer this question is via detailed comparisons between model predictions and exact numerical solutions of the Navier-Stokes equations for the full problem of a deformable bubble in the timeperiodic straining flow, given by (1). Such comparisons will be reported at the end of this paper. Here, however, we take a different tack. In particular, knowing that the full fluid mechanics problem exhibits a double-zero eigenvalue at the critical point, we can use the general unfolding theory for such a critical point to determine the form of the two-parameter dynamical model that exhibits the most general possible dynamical response, at least in the vicinity of the critical point. Thus, by comparison with (7), we can determine whether the form of our ad hoc model is sufficiently general to capture the expected dynamical response in the vicinity of a double-zeroeigenvalue critical point. Of course, the unfolding theory can only yield the expected form for the dynamical model near the critical point, and cannot yield any information about the coefficients of the various terms. For this, we must rely on the physically-based model equation, and on comparisons with solutions (numerical) of the exact problem.

A detailed discussion of the general unfolding theory for examination of dynamical behaviour in the vicinity of critical points is available elsewhere (cf. Guckenheimer \& Holmes). Here, we limit our discussion to the special case of the two-parameter unfolding of a double-zero-eigenvalue critical point.

Let us start or discussion by identifying two variables $x_{1}$ and $x_{2}$ as $x_{1}=x-x_{\mathrm{c}}$, and $x_{2}=\dot{x}_{1}$, where $x_{\mathrm{c}}$ is the measure of steady-state deformation at the critical point. Then, in general, the dynamics at the double-zero-eigenvalue critical point can be expressed in the form

$$
\left(\begin{array}{l}
\dot{x}_{1} \\
\dot{x}_{2}
\end{array}\right)=\left(\begin{array}{ll}
0 & 1 \\
0 & 0
\end{array}\right)\left(\begin{array}{l}
x_{1} \\
x_{2}
\end{array}\right)+o\left(\left|x_{1}\right|,\left|x_{2}\right|\right) \text {. }
$$

Now, however, it is known from normal form theory that there exists a smooth transformation $x_{1}=f\left(z_{1}, z_{2}\right)$ and $x_{2}=g\left(z_{1}, z_{2}\right)$, which can transform (8) into the form

$$
\left(\begin{array}{l}
\dot{z}_{1} \\
\dot{z}_{2}
\end{array}\right)=\left(\begin{array}{cc}
0 & 1 \\
0 & 0
\end{array}\right)\left(\begin{array}{l}
z_{1} \\
z_{2}
\end{array}\right)+\left(\begin{array}{c}
0 \\
a z_{1}^{2}+b z_{1} z_{2}
\end{array}\right)+o\left(\left|z_{1}\right|^{2},\left|z_{2}\right|^{2}\right) .
$$

Physically, $z_{1}$ in (9) can be considered as another suitable measure of deformation from the steady-state value. Finally, for the system (9), Takens (1974) proved that the local topological characteristic of any solution is completely determined by the linear and quadratic terms, i.e. by the truncated equation

$$
\left(\begin{array}{l}
\dot{z}_{1} \\
\dot{z}_{2}
\end{array}\right)=\left(\begin{array}{cc}
0 & 1 \\
0 & 0
\end{array}\right)\left(\begin{array}{l}
z_{1} \\
z_{2}
\end{array}\right)+\left(\begin{array}{c}
0 \\
a z_{1}^{2}+b z_{1} z_{2}
\end{array}\right)
$$


provided that $a \neq 0$. In other words, for non-vanishing $a(a \neq 0)$, the system (10) describes all possible dynamical behaviour at a double-zero-eigenvalue critical point. Finally, if $a \neq 0$, suitable scaling can reduce (10) further to the form

$$
\left(\begin{array}{l}
\dot{z}_{1} \\
\dot{z}_{2}
\end{array}\right)=\left(\begin{array}{ll}
0 & 1 \\
0 & 0
\end{array}\right)\left(\begin{array}{l}
z_{1} \\
z_{2}
\end{array}\right)+\left(\begin{array}{c}
0 \\
z_{1}^{2}+b z_{1} z_{2}
\end{array}\right)
$$

Now, we are interested in the dynamics near the critical point in the parameter space, for which the unfolding theory applies. For the two-parameter unfolding of (11), we have one of two possible cases: either

or

$$
\begin{aligned}
& \dot{z}_{1}=z_{2}, \quad \dot{z}_{2}=\mu_{1} z_{1}+\mu_{2} z_{2}+z_{1}^{2}+b z_{1} z_{2} \\
& \dot{z}_{1}=z_{2}, \quad \dot{z}_{2}=\mu_{1}+\mu_{2} z_{2}+z_{1}^{2}+b z_{1} z_{2},
\end{aligned}
$$

where $\mu_{1}$ and $\mu_{2}$ are parameters that are zero at the critical point. Thus, all possible dynamical features in the vicinity of a codimension- 2 , double-zero-eigenvalue critical point can be described by one of the forms (12) or (13). If there is no damping $\left(\mu_{2}=\right.$ $0)$, the dynamical system for many physical situations is Hamiltonian. In that case, the coefficient $b$ in (12) or (13) vanishes for $\mu_{2}=0$. Although the form (12) can be written in the form (13) by defining $z_{1}=z_{1}+\frac{1}{2} \mu_{1}$, the result is

$$
\dot{\tilde{z}}=z_{2}, \quad \dot{z}_{2}=\tilde{\mu}_{1}+\tilde{\mu}_{2} z_{2}+\tilde{z}_{1}^{2}+b \tilde{z}_{1} z_{2},
$$

where $\mu_{1} \equiv-\frac{1}{4} \mu_{1}^{2} \leqslant 0$. Thus, the coefficient $\tilde{\mu}_{1}$ is strictly negative (or zero), whereas $\mu_{1}$ in (13) may be either positive or negative. In fact, the forms (12) and (13) both correspond to important physical problems (see $\$ 8$ ), and it is useful to discuss both briefly, though our focus will be on the system (13) because it is directly relevant to the problem that is addressed by the present paper.

The system (12) has the special property that one of the two possible steady solutions is the trivial state $\left(z_{1}=z_{2}=0\right)$ for all values of $\mu_{1}$ and $\mu_{2}$, while a second steady solution is $z_{1}=-\mu_{1}, z_{2}=0$. Thus, the critical point for (12) is a transcritical bifurcation point for variable $\mu_{1}$ with fixed $\mu_{2}$. Two steady solutions exist for all $\mu_{1}$ and $\mu_{2}$, but there is an exchange of stability from one branch to the other at the critical point $\left(\mu_{1}=\mu_{2}=0\right)$. A number of important problems involving bubbles and drops exhibits this type of qualitative behaviour (see $\$ 8$ ). For example, the dynamical equation for an inviscid, charged drop near the Rayleigh limit, was shown by Tsamopoulos, Akylas \& Brown (1985) using a singular perturbation analysis to be exactly of the form (12) with vanishing $b$.

For the system (13), the critical point is a limit point. In particular, there are two steady solutions for $\mu_{1}<0$, but no solutions for $\mu_{1}>0$. The general phase-plane portrait of the solutions of (13) as a function of $\mu_{1}$ and $\mu_{2}$ is outlined by Guckenheimer \& Holmes (1983), and will not be repeated here. The term $\mu_{2} z_{2}$ acts as a 'viscous' damping if $\mu_{2}<0$. Further, in general, the term $b z_{1} z_{2}$ plays a role in the existence of limit cycles for some regions of the parameter space. In many physical problems, however, the dynamics is Hamiltonian in the absence of damping $\left(\mu_{2}=0\right)$, i.e. $b=$ 0 when $\mu_{2}=0$, and $b$ varies smoothly with $\mu_{2}$. In this case, the term $b z_{1} z_{2}$ does not have any significant effect on the qualitative dynamics described by (13), and all possible dynamical features are captured by the simpler system of equations with the term $b z_{1} z_{2}$ deleted, i.e.

$$
\dot{z}_{1}=z_{2}, \quad \dot{z}_{2}=\mu_{1}+\mu_{2} z_{2}+z_{1}^{2} .
$$

To see that this is true, we note that the Hopf and homoclinic bifurcation curves that generally exist in the $\left(\mu_{1}, \mu_{2}\right)$ space for the system (13), actually coincide when $b$ goes 
to zero smoothly as $\mu_{2} \rightarrow 0$ and correspond to the half-line $\mu_{2}=0, \mu_{1}<0$. On the other hand, it is known that limit cycles exist only in the region bounded by the Hopf and homoclinic bifurcation curves. Taken together, these facts mean that systems with the property that $b \rightarrow 0$ when $\mu_{2} \rightarrow 0$ can only exhibit limit cycles for $\mu_{2}=0\left(\mu_{1}<0\right)$. Thus, for the physically meaningful case where $\mu_{2}<0$ (positive viscosity), no limit cycles can exist, and in this case the term $b z_{1} z_{2}$ can be neglected without any effect on the qualitative behaviour of the dynamical model (i.e. the simpler model (15) suffices to capture all possible dynamical behaviour in the vicinity of the double-zeroeigenvalue limit point).

The critical question, then, is whether the simple ad hoc dynamical model (7) has the generic features that are exposed by the unfolding theory described above. Here, we restrict our discussion to the constant-strain case $(\epsilon \delta=0)$. The linearized version of the model (7) has already been shown to have a double-zero eigenvalue at $(w=$ $1, \mu=0$ ) for $x_{\mathrm{s}}=1$. By defining new variables

$$
z_{1}=x-1 \text { and } z_{2}=\dot{z}_{1}=\dot{x}
$$

the model (7) is transformed to the form

$$
\dot{z}_{1}=z_{2}, \quad \dot{z}_{2}=(w-1)-\epsilon \mu z_{2}+z_{1}^{2} .
$$

Thus, if we put $\mu_{1}=w-1$ and $\mu_{2}=-\epsilon \mu$, the system (17) is identical to the general form (15) that was derived above from the general unfolding theory. It follows that the model, although ad hoc, is capable of describing all possible dynamical features of the original problem, at least in the vicinity of the critical point, provided, of course, that the description of deformation in terms of the single scalar $x$ is sufficient. We believe this is true for the axisymmetric flow problem considered here, and we shall provide some corroboration via direct comparison between model prediction and exact numerical solutions, as we have already stated.

Assuming that the model (17) describes the dynamics near a critical point, we can construct a qualitative phase-plane portrait, based upon this model and the linear stability results, for the bubble dynamics in a steady, uniaxial straining flow. The result is sketched in figure 3 . We note from these results that bubble breakup is possible even at subcritical Weber numbers if the initial conditions are sufficiently far from the stable steady solution (represented in figure 3 by the centre point at $\dot{x}=$ $x=0$ ). On the other hand, for $\mu>0$ (i.e. $\mu_{2}<0$ ), breakup will not occur if the initial condition is in the shaded region. The phenomena of bubble breakup at subcritical Weber numbers for an initially deformed shape was first observed from unsteady numerical solutions for the full fluid mechanics problem by Kang \& Leal (1987). For the inviscid, potential flow limit, the stable and unstable regions in phase space are separated by the homoclinic orbit (the 'separatrix') which passes through the unstable (hyperbolic) fixed point. As $W$ is increased toward $W_{c}$, the separation between the stable (elliptic) and unstable fixed point decreases (and so too does the area of 'stability' within the homoclinic orbit), until finally at $W=W_{\mathrm{c}}$ they coincide and the limiting orbit (only) is cusped. For $W>W_{e}$, all initial conditions are unstable.

Although the phase-plane portraits of figure 3 provide a systematic basis to explain all of the behaviour that we have seen previously for steady straining flows, including the sensitivity of stability to initial conditions etc., the focus here is on the bubble response to time-periodic perturbations of the basic straining flow. In this framework, the most important feature of our dynamical model is the existence of the homoclinic orbit for $\epsilon=0$ (zero viscosity and steady straining flow). This suggests that the introduction of a time-periodic modulation of the strain rate will induce a 

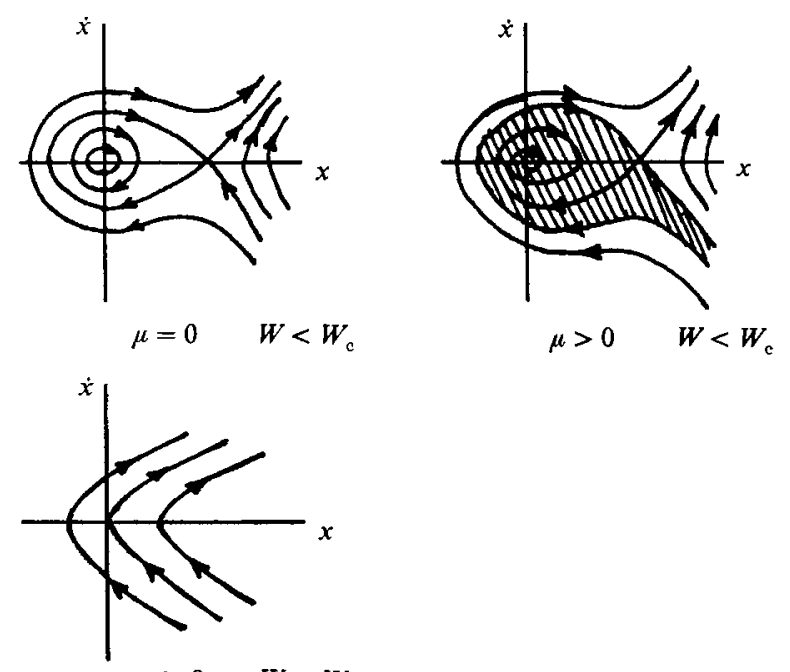

$\mu \geqslant 0 \quad W>W_{\mathrm{c}}$

Figure 3. Phase-plane representations of bubble dynamics in a steady, uniaxial straining flow.

transition from regular to chaotic behaviour. In the following section, we discuss a number of interesting dynamical features of the model equation, such as the existence of a resonant amplification in the shape oscillation at a critical frequency, and the possibility of bubble breakup at subcritical Weber number via chaotic oscillations of shape. Later, in $\S 7$, we shall present unsteady numerical solutions for the full fluid mechanics problem to corroborate the results from the dynamical model analysis. Finally, in the last section, we briefly discuss the applicability of the same dynamical model to a number of different physical problems that involve timedependent deformations of a bubble (or drop).

\section{Analyais of the dynamical model equation}

Let us begin this section by representing the model equation in a standard form. We define $y=x_{\mathrm{us}}-x, q=y$, and $p=\dot{y}$, where $x_{\mathrm{us}}$ represents the unstable steadystate solution for the unperturbed system $(\epsilon=0)$. Then, (7) can be expressed as

$$
\begin{aligned}
& \dot{q}=p, \\
& \dot{p}=\omega_{0}^{2} q-q^{2}-\epsilon(\mu p-\delta \cos \omega t),
\end{aligned}
$$

where $\omega_{0}^{2}=2(1-w)^{\frac{1}{2}}$. For the unperturbed case $(\epsilon=0)$, the system $(18)$ is a Hamiltonian system with a saddle point at the origin and a centre (or elliptic point) at $q=\omega_{0}^{2}$ (in the Poincaré map, they are fixed points). The frequency of oscillation near the centre is $\omega_{0}$.

Now we are interested in cases where $\epsilon \neq 0$. If $\epsilon \ll 1$ and $\mu=0$, almost all of the closed curves in the unperturbed Poincare map are preserved according to the KAM theorem. Especially, this is the case near the elliptic fixed point, so let us start our discussion with the regular closed orbital motion near this point.

\subsection{Regular motion near the centre}

In this section we analyse the dynamical behaviour of (18) near the elliptic fixed point via classical perturbation techniques, and compare the results with numerically 
constructed Poincaré maps. To do this, we shift the centre of $(18)$ to the origin by introducing $u=q-\omega_{0}^{2}$, so that (18) becomes

$$
\ddot{u}+(\epsilon \mu) \dot{u}+\omega_{0}^{2} u+u^{2}=\epsilon \delta \cos \omega t .
$$

In (19), we note that $\omega_{0}$ is the intrinsic frequency of the unperturbed system near the elliptic fixed point and $\omega$ is the frequency of the time-periodic forcing. If $\omega$ is far enough from $\omega_{0}$, no interesting result is expected at least up to the leading order of approximation (i.e. the $O(\epsilon)$ forcing yields only a $O(\epsilon)$ output for $u$ ). Thus, our main emphasis is given to the resonant case.

\subsubsection{Resonant case $\left(\omega=\omega_{0}\right)$}

If $\omega$ equals $\omega_{0}$, the linear approximation of (19) based upon the assumption that $u=O(\epsilon)$, does not have a bounded solution for $\mu=0$. Therefore, it is quite interesting to see if a bounded periodic solution for (19) can actually exist. To this end, the twotiming method (cf. Kevorkian \& Cole 1981) provides a powerful tool, and the leadingorder solution is found to be (for simplicity, we assume that $\delta=1$ for the following analyses)

$$
u(t) \sim \epsilon^{\frac{1}{3}} R(\tau) \cos (\omega t-\phi(\tau)),
$$

where $\tau=\epsilon^{\frac{2}{3}} t$, and $R(\tau), \phi(\tau)$ satisfy the set of equations

$$
\frac{\mathrm{d} R}{\mathrm{~d} \tau}=-\frac{1}{2}\left(\epsilon^{\frac{1}{3}} \mu\right) R+\frac{\sin \phi}{2 \omega}, \quad R \frac{\mathrm{d} \phi}{\mathrm{d} \tau}=\frac{5 R^{3}}{12 \omega^{3}}+\frac{\cos \phi}{2 \omega} .
$$

The fixed point of (21) is determined from the pair of equations

$$
\left(\epsilon^{\frac{1}{3}} \mu\right) \omega R=\sin \phi, \quad \frac{5 R^{3}}{6 \omega^{2}}=-\cos \phi .
$$

For $\mu=0$, we can easily show that the long-timescale problem (21) is a Hamiltonian system with Hamiltonian

$$
H=\frac{5 R^{4}}{48 \omega^{3}}+\frac{R \cos \phi}{2 \omega}
$$

and that it has a fixed point at $(R, \phi)=\left(\left(\frac{6}{5} \omega^{2}\right)^{\frac{1}{3}}, \pi\right)$. If we define $X=R \cos \phi$ and $Y=$ $R \sin \phi$, then the solutions of (21) for $\mu=0$ form closed orbits near the fixed point $\left(-\left(\frac{6}{5} \omega^{2}\right)^{\frac{1}{3}}, 0\right)$ in the $(X, Y)$-plane. If $\mu>0$, on the other hand, the fixed point determined by (22) is a stable attractor in the $(X, Y)$-plane, which indicates that the asymptotic behaviour of the solution (20) for $\mu>0$ is

$$
u \rightarrow \epsilon^{\frac{1}{3}} R_{\infty} \cos \left(\omega t-\phi_{\infty}\right) \text { as } t \rightarrow \infty,
$$

where $\left(R_{\infty}, \phi_{\infty}\right)$ is the solution of (22) for $\mu>0$. This equation shows that the longtime stable attractor corresponds to a limit cycle in the phase plane. Thus, this kind of resonant excitation may be used to generate a stable limit cycle for the non-zeroviscosity case.

One of the useful features of the two-timing solution is that it provides the Poincaré map directly. For example, the Poincaré map for (19) is simply obtained by substituting $t=n T$ in (20), where $T$ is the period of time-periodic forcing, i.e. $T=$ $2 \pi / \omega$. In terms of original variables, $q$ and $p$, the Poincaré map is given by

$$
\begin{gathered}
q(n T)\left(=u(n T)+\omega_{0}^{2}\right) \sim \epsilon^{\frac{1}{3}} R\left(\tau_{n}\right) \cos \left(\phi\left(\tau_{n}\right)\right)+\omega_{0}^{2}, \\
p(n T)(=\dot{u}(n T)) \sim \epsilon^{\frac{1}{2}} \omega R\left(\tau_{n}\right) \sin \left(\phi\left(\tau_{n}\right)\right),
\end{gathered}
$$




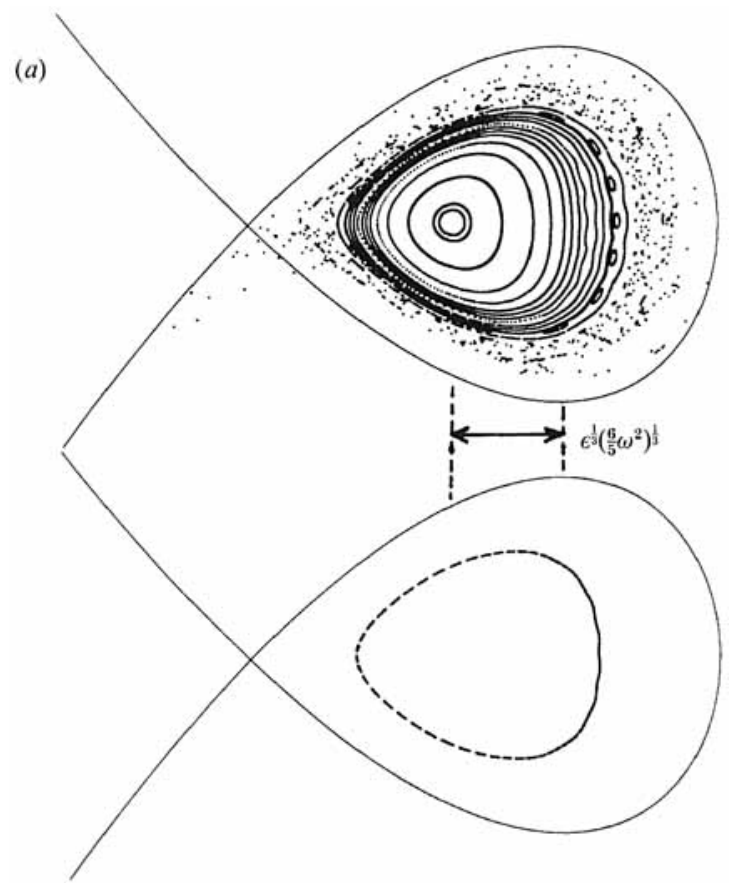

(b)

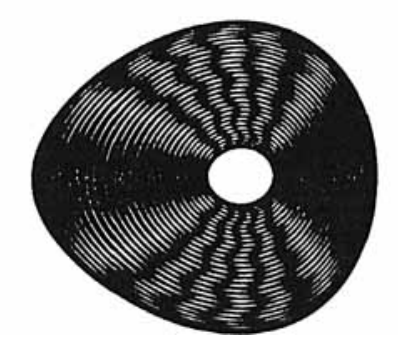

Figure 4. (a) A Poincaré map and $(b)$ a phase-plane flow for resonant forcing $\left(\omega_{0}=\omega=1, \epsilon=0.03, \delta=1, \mu=0\right)$.

where $\tau_{n}=\epsilon^{\frac{2}{3}} n T$. Since $q=\epsilon^{\frac{1}{3}} X+\omega^{2}$ and $p=\epsilon^{\frac{1}{3}} Y$, if $\mu=0$ the Poincaré map for (19) also has closed curves near the fixed point, and if $\mu>0$ the fixed point is a stable attractor that corresponds to a limit cycle in the phase plane.

The solution (20) is one of the most important results in the present paper. First of all, we see that the resonant interaction produces an $O\left(\epsilon^{\frac{1}{3}}\right)$ output from the $O(\epsilon)$ forcing. To demonstrate the resonance effect, one example of the numerically constructed Poincaré maps for the model system (19) is presented in figure 4. As predicted from the perturbation analysis, the fixed point is shifted to the left by an amount $\epsilon^{\frac{1}{3}}\left(\frac{6}{5} \omega^{2}\right)^{\frac{1}{3}}$ and there are closed orbits near the fixed point, which corresponds to the fact that $\mu=0$. The lower part of figure $4(a)$ is one specific closed curve in the Poincaré map, and the figure $4(b)$ is the corresponding trajectory in the phase plane (which demonstrates the bandwidth of the actual motion about the closed trajectory of the Poincaré map). 


\subsection{2. $n=2$ resonant case $\left(\omega=2 \omega_{0}\right)$}

As mentioned earlier, if $\omega$ is far from $\omega_{0}$, no particularly interesting results are expected near the centre, at least up to the leading-order approximation of the solution. In fact, the leading-order solution is simply

$$
u \sim \epsilon R \cos \left(\omega_{0} t-\phi\right)+\frac{\epsilon}{\omega_{0}^{2}-\omega^{2}} \cos \omega t,
$$

where $R$ and $\phi$ are constants that must be determined from the initial conditions. Thus, an $O(\epsilon)$ response is obtained from the $O(\epsilon)$ forcing. However, if we consider the next-order term, it is clear that there is again a multiple timescale response for the cases $\omega \sim 2 \omega_{0}$ and $\omega \sim \frac{1}{2} \omega_{0}$. In particular for these cases, the nonlinear term in (19) produces secular terms that must be suppressed in order to have bounded solutions at the second order of approximation. Since the $\omega=2 \omega_{0}$ case exhibits interesting behaviour, we briefly consider it below.

In the case $\omega=2 \omega_{0}$, we must have $R=0$ in order to suppress the secular term at second order if $R$ is assumed to be constant. However, with $R=0$ there is no way for the solution (25) to fit arbitrary initial conditions. Therefore we allow a longtimescale variation of $R$ and $\phi$ (i.e. the solution again exhibits a two-timescale structure can be analysed via the two-timing method). In fact, (25) is modified for the case $\omega=2 \omega_{0}$ to the form

$$
u \sim \epsilon R(\tau) \cos \left(\omega_{0} t-\phi(\tau)\right)-\frac{\epsilon}{3 \omega_{0}^{2}} \cos \left(2 \omega_{0} t\right),
$$

where $\tau=\epsilon t$. The functions $R(\tau)$ and $\phi(\tau)$ can be shown to satisfy the dynamical equations

$$
\frac{\mathrm{d} R}{\mathrm{~d} \tau}=\frac{R \sin 2 \phi}{6 \omega_{0}^{3}}, \quad \frac{\mathrm{d} \phi}{\mathrm{d} \tau}=\frac{\cos 2 \phi}{6 \omega_{0}^{3}} .
$$

As expected, (27) has a fixed point at $R=0$, which can be easily seen to be a saddle point in the $(R \cos \phi, R \sin \phi)$-plane. Along the curves $\phi=\frac{1}{4} \pi$ and $\frac{5}{4} \pi, \mathrm{d} \phi / \mathrm{d} \tau=0$ and $\mathrm{d} R / \mathrm{d} \tau>0$, which means they are unstable manifolds. But along $\phi=\frac{3}{4} \pi$ and $\frac{7}{4} \pi$, $\mathrm{d} \phi / \mathrm{d} \tau=0$ and $\mathrm{d} R / \mathrm{d} \tau<0$, i.e. they are stable manifolds. We shall see later (figure 10) that the numerically constructed Poincaré map also exhibits a transition from an elliptic fixed point (or centre) for $\omega \approx \omega_{0}$ to a saddle-node fixed point for $\omega=2 \omega_{0}$. One point worth emphasizing, however, is that the strong resonance associated with the case $\omega=\omega_{0}$ does not occur for $\omega=2 \omega_{0}$. Here, the $O(\epsilon)$ force yields only an $O(\epsilon)$ response.

As in $§ 6.1 .1$, we can construct the Poincaré map near the centre of the unperturbed system by using the two-timing solution (26) and (27). In the Poincaré map we have

$$
\begin{aligned}
& q(n T) \sim(-1)^{n} \epsilon R\left(\tau_{n}\right) \cos \left(\phi\left(\tau_{n}\right)\right)-\frac{\epsilon}{3 \omega_{0}^{2}}+\omega_{0}^{2}, \\
& q(n T) \sim(-1)^{n} \epsilon \omega_{0} R\left(\tau_{n}\right) \sin \left(\phi\left(\tau_{n}\right)\right),
\end{aligned}
$$

where $T=2 \pi / \omega=\pi / \omega_{0}$, and $\tau_{n}=\epsilon n T$.

6.1.3. Boundedness of the regular region and the effect of the amplitude change of the time-periodic forcing

As we can see in figure 4, the regular region near the elliptic centre is bounded by a chaotic region, where many dots of the Poincaré map are distributed in a more or 

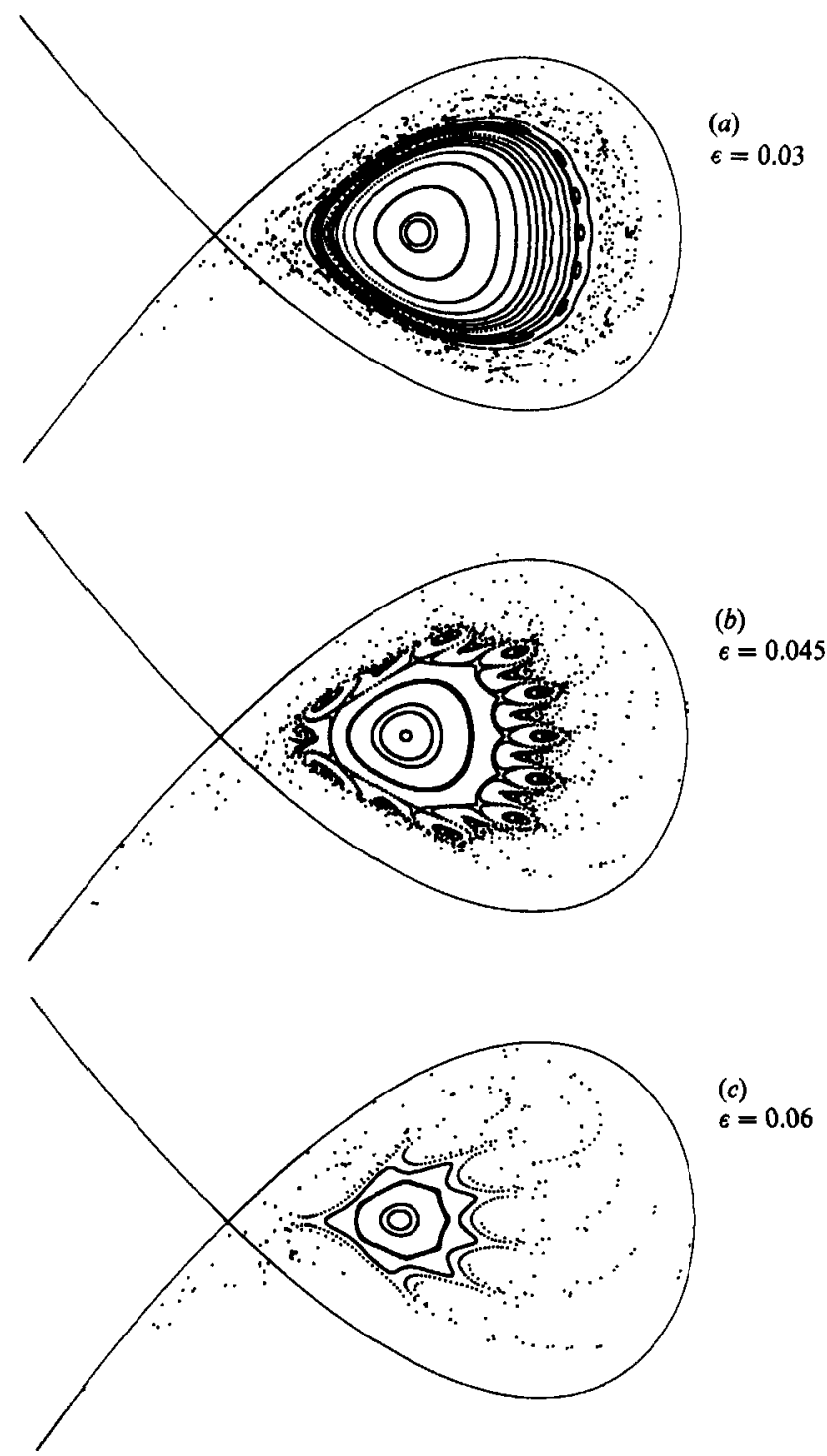

Fraure 5. The effect of the forcing amplitude on the Poincaré map $\left(\omega_{0}=\omega=1, \delta=1, \mu=0\right)$.

less random manner. In the following section, we shall discuss chaotic bubble motion and breakup in time-periodic straining flows. Here we discuss the effect of changes in the amplitude of the time-periodic forcing on the size of the regular region.

For the case of resonant forcing $\left(\omega=\omega_{0}=1\right)$, three Poincaré maps are given in figure $5(a-c)$ for $\epsilon=0.03,0.045$, and 0.06 with other parameters fixed $(\delta=1, \mu=0)$. As expected, the regular region shrinks in size as the forcing amplitude increases. Thus, some initial conditions may be in the chaotic region for larger forcing amplitudes, while the same initial conditions are in the regular region for smaller amplitudes. For example, let us consider the point $(q, p)=(1,0)$ that is the centre for the unperturbed case $(\epsilon=0)$. In the case $\epsilon=0.03$, the point $(1,0)$ is in the regular region and the point is mapped to the points on the closed curve that includes the point $(1,0)$ (figure $5 a$ ). When $\epsilon$ is increased to 0.045 , the point $(1,0)$ is in the 


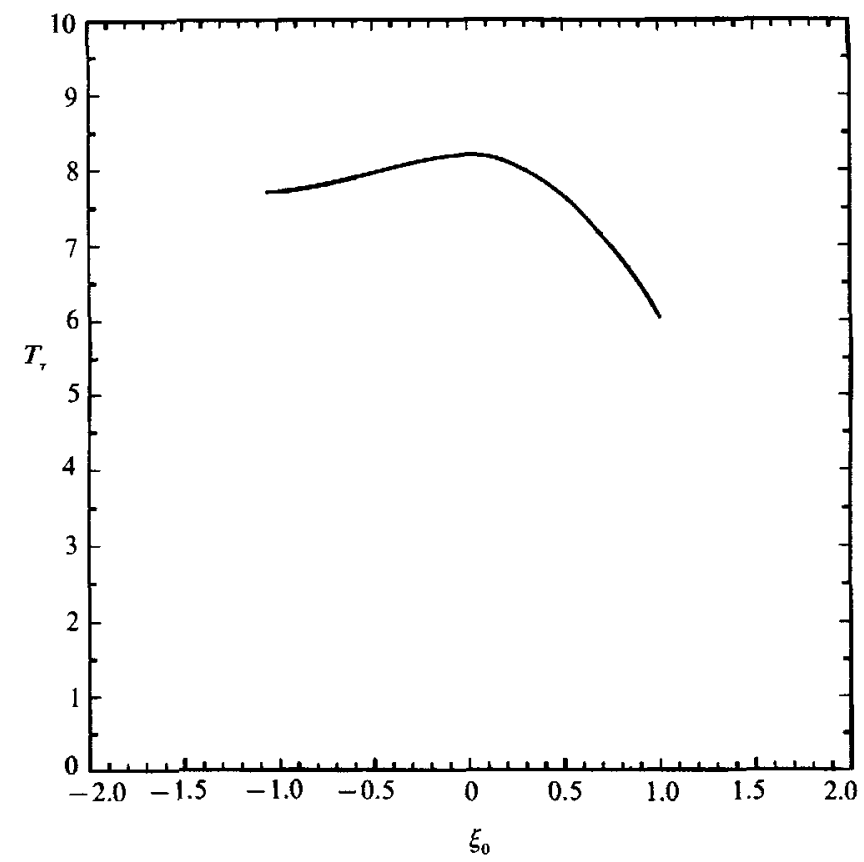

Figure 6. Period of the system (21) for $\mu=0$ as a function of initial condition $\left(\xi_{0}, 0\right)$.

transitional structure that is between the regular and the chaotic regions (figure $5 b$ ). Finally, for $\epsilon=0.06$, the point $(1,0)$ is in the chaotic region, and the point is mapped to other points in the chaotic region in a non-regular manner up to a certain iteration, until eventually the point is mapped to the breakup region that lies outside the separatrix of the undisturbed system (see the detailed discussion in the following section).

In figure 5, we can also see several islands in the Poincaré maps that are closed curves around $k$-periodic points (11-periodic points for $\epsilon=0.03$, and 9-periodic points for $\epsilon=0.045$ ). In order to understand how such a large number of periodic points are obtained, let us look at the system of equations (21). For $\mu=0,(21)$ is a Hamiltonian system and its solutions form closed curves near the fixed point. Now let us denote the period in $\tau$ of the orbit in the phase plane, $(\xi, \eta)=(R(\tau) \cos \phi(\tau)$, $R(\tau) \sin \phi(\tau))$, by $T_{r}$. The period of the closed orbit $\left(T_{\tau}\right)$ starting at $\left(\xi_{0}, 0\right)\left(\xi_{0}>-\left(\frac{6}{5}\left(\omega^{2}\right)^{\frac{1}{3}}\right)\right.$ is plotted in figure 6 for the case when $\omega=\omega_{0}=1$. If a certain point on the closed orbit in the $(\xi, \eta)$-plane corresponds to a $k$-periodic point in the Poincaré map, then one cycle in the $(\xi, \eta)$-plane corresponds to exactly one cycle in the Poincare map (see equation (24)). However, for each cycle, it takes a period $T_{\tau}$ in terms of $\tau$ in the ( $\xi$, $\eta$ )-plane, while it takes a period $k T$ in terms of $t$ in the Poincaré map (because it is a $k$-periodic point). Furthermore, since we have $\tau=\epsilon^{\frac{2}{3} t}$, the relation between $T_{\tau}$ and the period of forcing $T$ is given by

Therefore

$$
\begin{gathered}
T_{\tau}=\epsilon^{\frac{2}{3}} k T=\epsilon^{\frac{2}{3}} k\left(\frac{2 \pi}{\omega}\right) . \\
k=\epsilon^{-\frac{2}{3}}\left(\frac{\omega}{2 \pi}\right) T_{\tau} .
\end{gathered}
$$

For example, $k=11$ is obtained from $T_{\tau}=6.67$ for the case $\omega=1$, and $\epsilon=0.03$, while $k=9$ is obtained from $T_{\tau}=7.15$ for $\omega=1$, and $\epsilon=0.045$. From figure 6 , we can see 
(a)

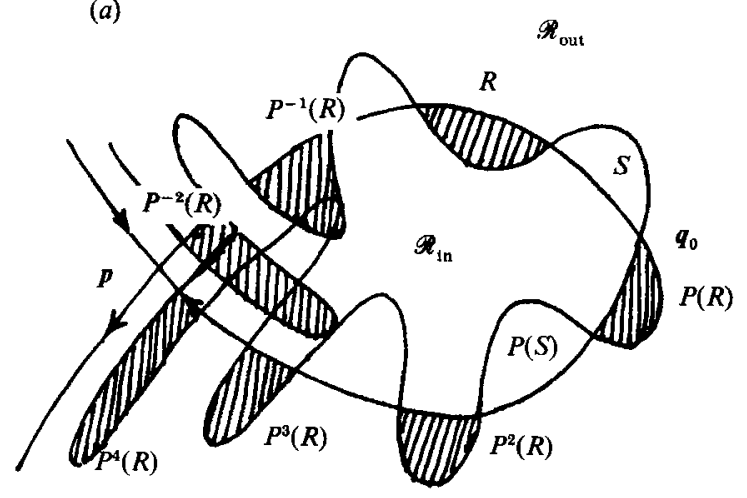

(b)

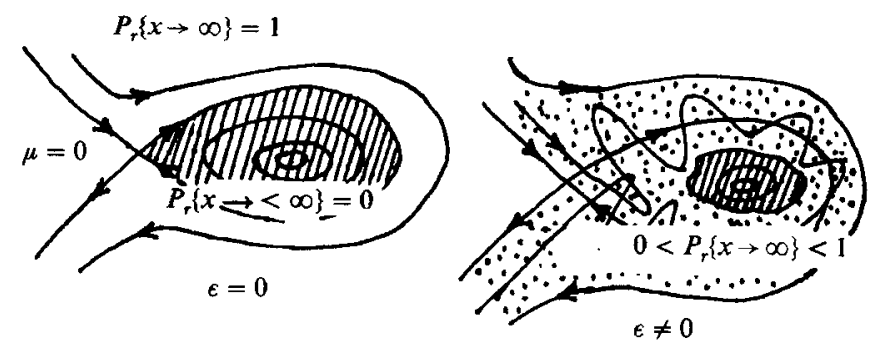

(c)

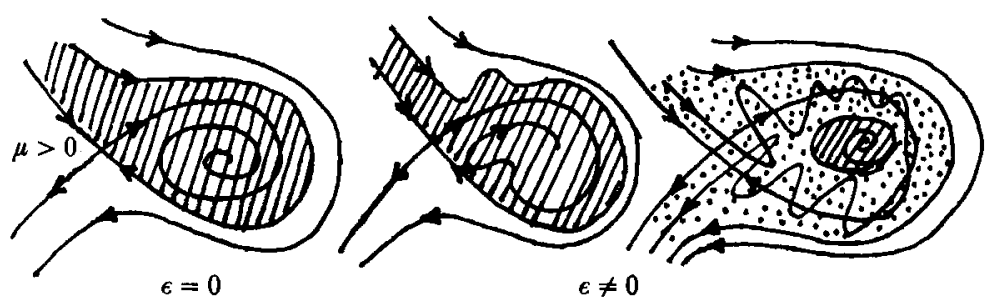

Figure 7. A schematic representation of a homoclinic tangle and the relationship to bubble breakup.

that $T_{\tau}$ decreases monotonically from 8.18 to 6.15 as $\xi_{0}$ increases from 0 to 1 . Here we should note that the values 6.67 and 7.15 are in the monotonically decreasing range of $8.18>T_{\tau}>6.05$. Since $T_{\tau}$ is decreasing monotonically, the angular velocity near the $k$-periodic points is higher for larger $\xi_{0}$, and lower for smaller $\xi_{0}$. The difference in the angular velocity makes a closed curve in the Poincare maps.

\subsection{Chaotic bubble motion and breakup}

Chaotic motion resulting from homoclinic orbit tangling for a perturbed twodimensional map is well explained elsewhere (cf. Guckenheimer \& Holmes 1983 or Wiggins 1988), so here we touch only on the effect of time-periodic forcing on bubble breakup in terms of homoclinic tangling.

Let us consider figure $7(a)$, which shows that the homoclinic orbit breaks into an unstable manifold (denoted by $W_{\mathrm{u}}(\boldsymbol{p})$ ) and a stable manifold (denoted by $W_{\mathrm{s}}(\boldsymbol{p})$, which form a tangle. In figure 7 , the point $p$ is the hyperbolic fixed point and $q_{0}$ is the primary intersection point of the unstable and the stable manifolds. For a 
discussion of bubble breakup in terms of two-dimensional Poincaré maps, let us first summarize some general properties of such maps without any detailed discussion (for discussion, see Guckenheimer \& Holmes or Wiggins).

(p1) A stable (or unstable) manifold cannot intersect itself, but it may intersect an unstable (or stable) manifold.

(p2) If there is an intersection of the unstable and the stable manifolds, then there are infinitely many intersections.

(p3) If the system is Hamiltonian, the map preserves the area, for example, the map $P: P^{k}(R) \rightarrow P^{k+1}(R)$ preserves the area of the region between the stable and unstable manifolds.

Now we can use the boundary of the closed curve $\left(W_{\mathrm{u}}\left(\boldsymbol{p} \boldsymbol{q}_{\mathbf{0}}\right) \cup W_{\mathrm{s}}(\boldsymbol{q} \circ \boldsymbol{p})\right)$ to divide the two-dimensional domain into an internal region $\left(\left(\mathscr{\mathscr { T }}_{1 n}\right)\right.$ and an external region $\left(\mathscr{R}_{\text {out }}\right)$. To understand the significance of $\mathscr{R}_{\text {in }}$ and $\mathscr{R}_{\text {out }}$, let us first consider the unperturbed case. In the unperturbed case, the closed curve is the homoclinic orbit and bubble breakup is impossible for a bubble that is initially in $\mathscr{A}_{\text {in }}$ (see figure 2 ) (i.e. bubble breakup is possible only if a bubble is in $\mathscr{R}_{\text {out }}$ ). However, breakup is possible for the perturbed case, and we are interested in the possibility of bubble breakup for a bubble that is initially in $\mathscr{R}_{\mathrm{n}}$. The phenomenon can be well understood on the basis of the following three facts. First, if a bubble is initially in the region $P(R)$ it will surely break up eventually, because the maps for the following periodic times $(t=$ $k T)$ are

$$
P(R) \rightarrow P^{2}(R) \rightarrow \ldots \rightarrow P^{k}(R) \rightarrow \ldots
$$

and $P^{k}(R)$ will be extended indefinitely as $k \rightarrow \infty(\mathrm{by}(\mathrm{p} 2)$ and (p3)). Second, the only possible way for a bubble from the inside region to be transported to the outside region is via the map $R \rightarrow P(R)$, and the way to transport a bubble from outside to inside is via the map $S \rightarrow P(S)$. Therefore, the most important information for the exchange efficiency between $\mathscr{R}_{\mathrm{in}}$ and $\mathscr{R}_{\text {out }}$ is the areas of the regions $P(R)$ and $P(S)$. Third, the transportation within $\mathscr{R}_{\text {in }}$ is done by the map

$$
\ldots \rightarrow P^{-m}(R) \rightarrow \ldots \rightarrow P^{-1}(R) \rightarrow R .
$$

Here we must note that as $m \rightarrow \infty$ the structure of $P^{-m}(R)$ becomes extremely complicated, because by $(\mathrm{p} 2)$ and $(\mathrm{p} 3), P^{-m}(R)$ should be extended indefinitely, and by $(\mathrm{p} 1)$ the extended $P^{-m}(R)$ must lie entirely inside the region surrounded by the stable manifold $\left(W_{\mathrm{s}}(p)\right)$. This means that the chaotic region becomes subdivided, at an increasingly fine scale, between points that arrive within the region $R$ after (or prior to) $m$ periods, and points that do not. Although an exact determination of the fate of some arbitrary given initial point is possible in principle via numerical integration of the dynamical equations, if the goal of theory is to understand the overall rate of bubble breakup, we should attempt to develop a probabilistic description of the breakup of bubbles that are initially in $\mathscr{R}_{\text {in }}$. As suggested by the qualitative sketch in figure $7 b$, the probability of breakup in the steady inviscid flow $(\mu=0, \epsilon=0)$ is unity if the initial point lies outside the separatrix, but zero if it lies inside. On the other hand, when $\epsilon \neq 0$, the phase plane is subdivided into three zones: one inside the remaining regular region (which is much reduced in size) where $P_{r}(x \rightarrow \infty)=0$; one outside the chaotic zone where $P_{r}(x \rightarrow \infty)=1$; and the third within the chaotic region where $0<P_{r}(x \rightarrow \infty)<1$. The last statement must be understood as an averaged probability for all points within the chaotic zone; the probability of breakup for any specific point is either zero or one, but points with probability zero and one may lie arbitrarily close to one another in this region. The case $\mu>0$, sketched in figure $7(c)$, will be discussed shortly. 
First, however, let us attempt to estimate the probability that a bubble that is initially in the region $\mathscr{R}_{\text {in }}$ will be pumped out to $\mathscr{R}_{\text {out }}$ after $n$-iterations. The basic idea is the same as the detailed treatment of Rom-Kedar (1988) for transport of a tracer in two-dimensional maps. Although her treatment is rigorous, it requires a great deal of computation to get any information. Therefore, we attempt to estimate the probability in a very simple approximate manner in order to gain qualitative insight into the effect of time-periodic forcing on bubble breakup. Let us first introduce some notation:

$$
\begin{array}{ll}
I=\text { area of the region } \mathscr{R}_{\mathrm{n}} ; & C=\text { area of the chaotic region in } \mathscr{A}_{\mathrm{n}} ; \\
S=\text { area of the region } S ; & R=\text { area of the region } R,
\end{array}
$$

and the following assumptions:

(a1) The region $\mathscr{R}_{\mathrm{n}}$ consists of the disjoint regular and chaotic regions, i.e. $\mathscr{B}_{\mathrm{n}}=$ $\mathscr{R}_{\text {reg }} \cup \mathscr{R}_{\text {cha }}$ and $\mathscr{R}_{\text {reg }} \cap \mathscr{R}_{\text {cha }}=\phi$.

(a2) If a bubble is initially in the regular region, the bubble never breaks up.

(a3) At each iteration, the probability that a bubble is pumped out to the region $\mathscr{R}_{\text {out }}$ is assumed to have the same value for all points in $\mathscr{R}_{\text {cha }}$; in other words, we are interested in the area-averaged probability over the region $\mathscr{R}_{\text {cha }}$.

(a4) We restrict our attention to the Hamiltonian system.

Now, let us introduce notation for several conditional probabilities:

$$
\begin{aligned}
P_{\text {out }, n} & =\operatorname{Pr}\left\{(q(n T), p(n T)) \in \mathscr{R}_{\text {out }} \mid(q(0), p(0)) \in \mathscr{R}_{\text {in }}\right\} \\
P_{\text {cha }, 0} & =\operatorname{Pr}\left\{(q(0), p(0)) \in \mathscr{R}_{\text {cha }} \mid(q(0), p(0)) \in \mathscr{R}_{\mathrm{nn}}\right\} \\
Q_{\text {cha }, n} & =\operatorname{Pr}\left\{(q(n T), p(n T)) \in \mathscr{R}_{\mathrm{cha}} \mid(q(0), p(0)) \in \mathscr{R}_{\mathrm{cha}}\right\}
\end{aligned}
$$

In other words, $P_{\text {out, } n}$ is the probability that a point initially in $\mathscr{R}_{1 \mathrm{n}}$ is in $\mathscr{R}_{\text {out }}$ at iteration $n$ (which is equivalent to being pumped out before or at the $n$th iteration because once a point is pumped out it never returns to $\mathscr{R}_{\mathrm{n}}$ again), $\boldsymbol{P}_{\text {cha,0 }}$ is the probability that a point initially in $\mathscr{R}_{\text {in }}$ is also in $\mathscr{R}_{\text {cha }}$, and $Q_{\text {cha, } n}$ is the probability that a point initially in $\mathscr{R}_{\text {cha }}$ remains in $\mathscr{R}_{\text {cha }}$ at iteration $n$. Then, we can easily see that by the assumption (a2)

$$
P_{\text {out }, n}=P_{\text {cha, },}\left(1-Q_{\text {cha, } n}\right) \text {. }
$$

Also, from the assumption (a3), we can see that

$$
Q_{\mathrm{cha}, n}=Q_{\mathrm{cha}, n-1}\left(1-\frac{R}{C}\right), \quad \text { i.e. } Q_{\mathrm{cha}, n}=\left(1-\frac{R}{C}\right)^{n}
$$

Finally, if the initial distribution is assumed to be uniform in $\mathscr{R}_{\mathrm{n}}$, then by the assumption (a1)

$$
P_{\mathrm{cha}, 0}=C / I \text {. }
$$

Therefore the final expression for $P_{\text {out, } n}$ is

$$
P_{\text {out }, n}=\frac{C}{I}\left\{1-\left(1-\frac{R}{C}\right)^{n}\right\}
$$

It is quite interesting to see the effect of variations of the areas of the regions $R$ and $\mathscr{R}_{\text {cha }}$. First we can see that the probability of bubble breakup is increased as the area of the region $R$ increases, i.e.

$$
\frac{\partial P_{\text {out }, n}}{\partial R}=\frac{n}{I}\left(1-\frac{R}{C}\right)^{n-1}>0
$$


In order to see the effect of the size of the chaotic region, let us represent (28) in the alternative form

$$
P_{\text {out, } n}=R / I\left(1+x+x^{2}+\ldots+x^{n-1}\right),
$$

where $x=1-R / C$. Since $x>0$ and $\partial x / \partial C>0, \partial P_{\text {out, } n} / \partial C>0$, i.e. the probability of bubble breakup increases as the area of the chaotic region increases.

For the case of $\mu=0$, the Poincaré map of (18) always has a homoclinic tangle, but if $\mu>0$ a homoclinic tangle (i.e. chaotic bubble motion) is not always possible. In order to have a homoclinic tangle, the ratio of the amplitude of forcing to the viscosity must exceed a certain critical value, which is a function of the forcing frequency. The situation is presented schematically in figure $7(c)$. For $\epsilon=0$, the stable fixed point is an attractor, and the stable and unstable manifolds that emanate from the hyperbolic fixed point do not intersect. Thus, when a weak time-periodic perturbation is added to the straining flow, the stable and unstable manifolds are distorted but they still do not intersect and the motion remains regular throughout the phase plane. However, as the amplitude of the perturbation increases, it is possible that the distorted unstable and stable manifolds eventually may intersect. If this happens, then by the property (p2), there will be infinitely many intersections and a homoclinic tangle occurs (i.e. the bubble motion becomes chaotic in the corresponding regions of the phase plane).

The condition for the chaotic bubble motion (or equivalently the existence of a homoclinic tangle) for $\epsilon \ll 1$ can be obtained analytically via the Melnikov function (cf. Guckenheimer \& Holmes). The Melnikov function essentially provides a measure of the separation between the stable and unstable manifolds in the phase plane. Hence, when the Melnikov function equals zero, the stable and unstable manifolds intersect. The condition for existence of a homoclinic tangle, obtained from setting the Melnikov function equal to zero, is

$$
\left(\frac{\delta}{\mu}\right) \geqslant \frac{\omega_{0}^{5} \sinh \left(\pi \omega / \omega_{0}\right)}{\pi \omega^{2}} \equiv R_{0}(\omega) .
$$

In general, an increase of the viscosity $\mu$ produces a stronger attractor, and thus increases the separation between stable and unstable manifolds, while an increase in the amplitude of the periodic forcing results in increased deformation of the manifolds and an increasing tendency toward intersection. The balance between these two effects is a very strong function of the forcing frequency as shown in (31). The limiting forms of $R_{0}(\omega)$ in (31) are

$$
\begin{array}{ll}
\text { as } \omega \rightarrow \infty & R_{0}(\omega) \propto \exp \left(\frac{\pi \omega}{\omega_{0}}\right), \\
\text { as } \omega \rightarrow 0 & R_{0}(\omega) \propto \frac{1}{\omega} .
\end{array}
$$

Therefore, in both extremes $R_{0}(\omega) \rightarrow \infty$. The minimum required forcing amplitude to produce chaotic motions occurs at the minimum of $R_{0}(\omega)$, i.e. at

$$
\omega_{\mathrm{opt}}=\frac{1.915 \omega_{0}}{\pi}
$$

In figure $8, R_{0}(\omega)$ is plotted for $\omega_{0}=1$ and we can see that $R_{0}(\omega)$ (and hence the critical forcing amplitude $\delta$ ) is a very strong function of $\omega$. For example, there is several orders-of-magnitude difference in the required forcing amplitudes between the cases $\omega=1$ and $\omega=5$. 


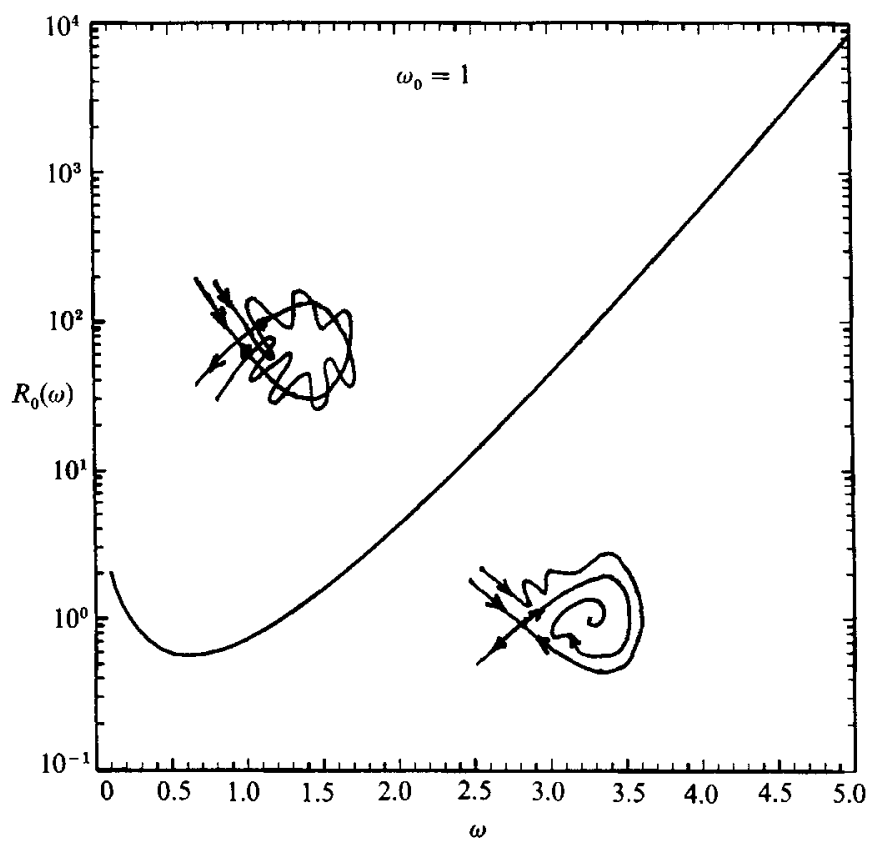

Figure 8. The condition for existence of a homoclinic tangle for $\mu>0$.

The function $R_{0}(\omega)$ can also be a good qualitative indicator of the exchange efficiency that we have considered by the maps $R \rightarrow P(R)$ and $S \rightarrow P(S)$. As discussed following (31), $R_{0}(\omega)$ can be thought of qualitatively as a measure of the difficulty in deforming the stable and unstable manifolds. Therefore,we can expect less deformation (i.e. smaller regions $R$ and $S$ ) as $R_{0}(\omega)$ increases for fixed $\delta$ and $\mu$ which satisfy the inequality (31). In order to see the effect of a change in the forcing frequency, the homoclinic tangles for several frequencies are shown in figure 9 for fixed values of $\omega_{0}, \epsilon, \delta$ and $\mu=0$. It should be noted that the area $P(R)$ decreases drastically as $\omega$ increases from 1 . The effect of the forcing frequency can also be examined from another point of view. In figure 10, the areas of the regular region (the region where the probability of bubble breakup is zero) are compared for different forcing frequencies with the intrinsic frequency $\omega_{0}=1$. As we can see in figure 10, the area of the regular region has a minimum near the resonant frequency, i.e. $\omega \approx \omega_{0}$. It should be noted in this regard that the case $\omega=0$ in figure 10 has a smaller $\epsilon$ by a factor of almost two compared to the other cases considered.

\subsection{Summary of the model analysis}

So far we have considered bubble dynamics in time-periodic straining flows via dynamic model analysis, and we have found the following facts.

(i) Near the stable steady solutions, an $O\left(\epsilon^{\frac{1}{3}}\right)$ dynamical output can be obtained from an $O(\epsilon)$ forcing by resonant amplification for $\omega \sim \omega_{0}$.

(ii) With other parameters fixed, the probability of bubble breakup at subcritical Weber numbers via chaotic oscillations of shape can be maximized by choosing the optimal forcing frequency, which is found to be close to the intrinsic frequency of the unperturbed system. 

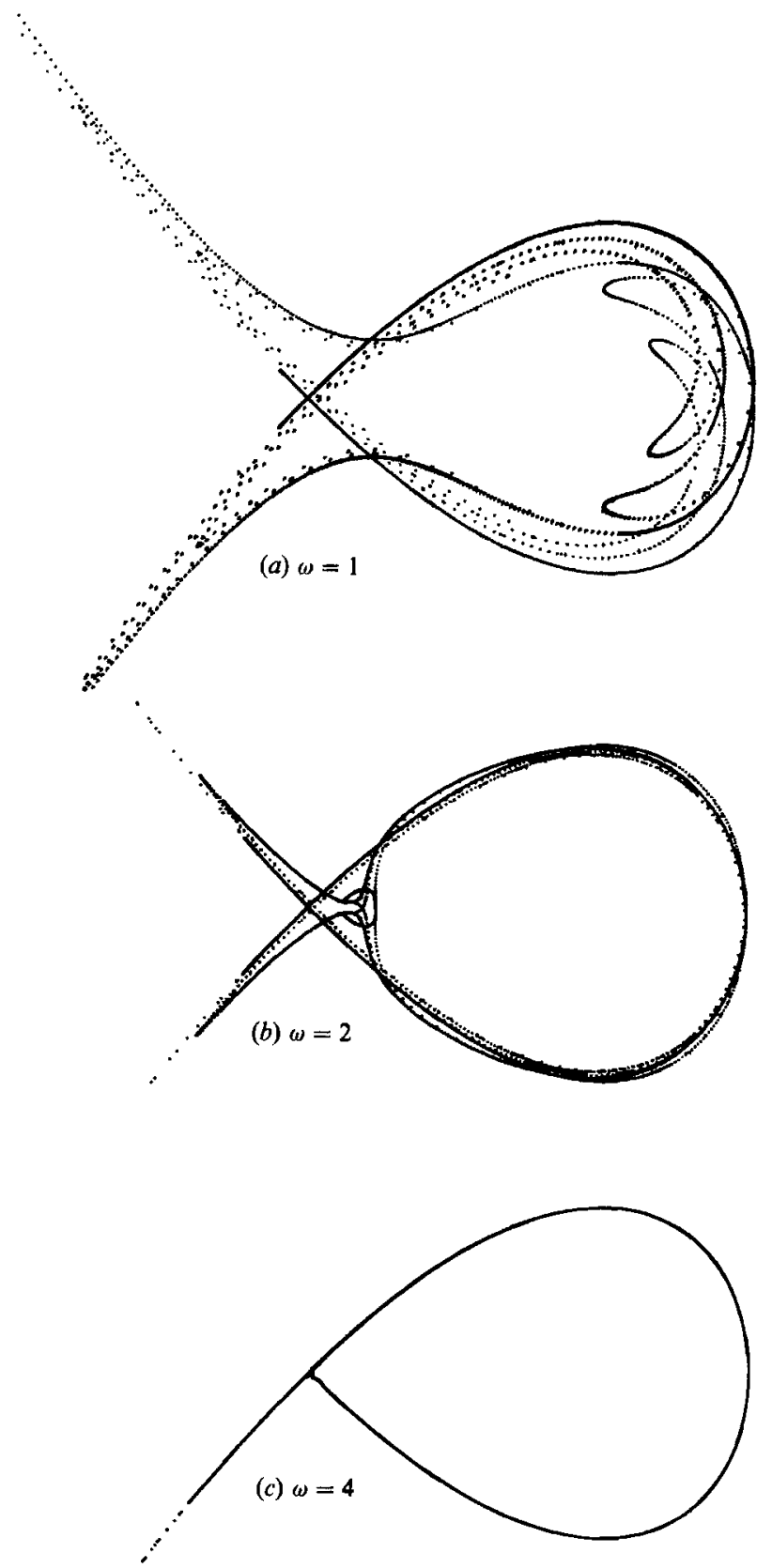

Figure 9. The effect of forcing frequency on a homoclinic tangle for $\omega_{0}=1, \epsilon=0.05, \delta=1$, $\mu=0$.

\section{Numerical results for the full fluid mechanisms problem}

In this section, we present a limited number of numerical results for the full fluid mechanics problem which corroborate the qualitative conclusions derived from the model analysis. In particular, we have calculated the dynamics of changes in bubble shape for time-periodic straining flows, using the numerical technique developed by 


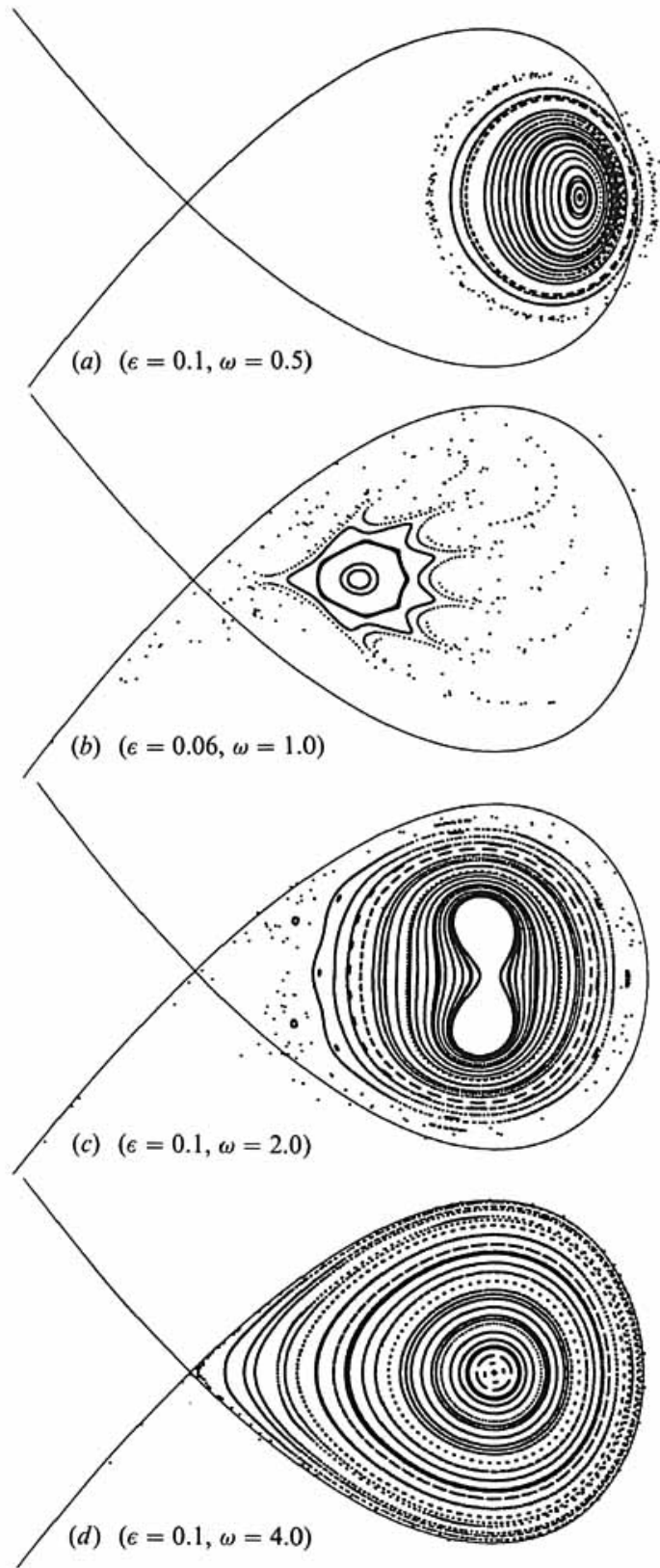

Figure 10. The effect of forcing frequency on the area of the region of regular motion for $\delta=1$ and $\mu=0$.

Kang \& Leal (1987). In our analysis, the effect of a time-periodic strain rate is characterized by a time-dependent Weber number that is defined by

$$
W(t)=\frac{2 \rho(E a)^{2} a}{\gamma}
$$

From (32), we can see that a time-periodic Weber number is obtained if the strain rate is time-periodic. However, that is not the only possibility. We may also consider 
the 'imaginary' situation of a time-periodic variation of surface tension. We can anticipate that the results will be similar to time-dependent changes in strain rate. The dynamic model in $\$ 4$ includes a time-periodic Weber number only, but cannot exhibit any difference between the two cases of time variations in $E$ or $\gamma$. Therefore, it is interesting to see if the same qualitative dynamical behaviour is obtained for these two cases when calculated from numerical solutions of the full fluid mechanics problem.

If the strain rate and surface tension are constant, of course, the dynamics is exactly the same for all combinations of strain rate and surface tension that yield the same Weber number, at least in the case of potential flow. However, this is not the case for a time-dependent strain rate or surface tension. The boundary conditions for these two problems are a little different. Therefore, we analyse the two cases separately for the same initial conditions to see if the difference in the boundary conditions result in any fundamentally different dynamical behaviour.

\subsection{The case of $\gamma(t)=\gamma(t+T)$ and $\dot{E}=0$}

For the case of a constant strain rate and time-periodic variations of surface tension, it is convenient to use the following characteristic scales:

$$
l_{\mathrm{c}}=a, \quad t_{\mathrm{c}}=E^{-1}, \quad U_{\mathrm{c}}=E a .
$$

Then the Navier Stokes equation for axisymmetric problems in terms of the boundary-fitted orthogonal coordinate system $(\xi, \eta)$ is given by

$$
\begin{gathered}
\frac{1}{2} R\left[\left(\frac{\partial \omega}{\partial t}\right)_{x, \sigma}+\frac{1}{h_{\eta} h_{\xi}}\left\{\frac{\partial \psi}{\partial \xi} \frac{\partial}{\partial \eta}\left(\frac{\omega}{\sigma}\right)-\frac{\partial \psi}{\partial \eta} \frac{\partial}{\partial \xi}\left(\frac{\omega}{\sigma}\right)\right\}\right]=\mathrm{L}^{2}(\omega \sigma), \\
\mathrm{L}^{2} \psi+\omega=0
\end{gathered}
$$

where $h_{\eta}$ and $h_{\xi}$ are the scale factors, and

$$
\mathrm{L}^{2} \equiv \frac{1}{h_{\eta} h_{\xi}}\left\{\frac{\partial}{\partial \xi}\left(\frac{f}{\sigma} \frac{\partial}{\partial \xi}\right)+\frac{\partial}{\partial \eta}\left(\frac{1}{f \sigma} \frac{\partial}{\partial \eta}\right)\right\} .
$$

We assume, for convenience, that the coordinate mapping is defined with $\xi=1$ corresponding to the interface, and with $\eta=0$ and $\eta=1$ being the symmetry axes. For boundary conditions, we require at the gas-liquid interface $(\xi=1)$

$$
\psi=\int_{0}^{\eta}\left(-u_{\xi}\right) \sigma h_{\eta} \mathrm{d} \eta=\int_{0}^{\eta}\left(-\frac{1}{|\nabla F|} \frac{\partial F}{\partial t}\right) \sigma h_{\eta} \mathrm{d} \eta,
$$

corresponding to the kinematic condition. In (35), $F$ is a function that describes the bubble shape as $F(x, t)=0$ and $u_{\xi}$ is the inward normal velocity. In addition, the vorticity at the bubble surface is given by

$$
\omega=\frac{2}{h_{\eta}} \frac{\partial u_{\xi}}{\partial \eta}+2 \kappa_{(\eta)} u_{\eta}
$$

corresponding to the condition of zero tangential stress (where $\kappa_{(\eta)}$ is the normal curvature of the interface in the $\eta$-direction and $u_{\eta}$ is the tangential velocity). Finally, the normal stress contributions due to pressure and viscous forces, on the one hand, and the capillary force, on the other, are required to balance

$$
\tau_{\xi \xi}-\frac{4}{W(t)}\left(\kappa_{(\eta)}+\kappa_{(\phi)}\right)=0
$$




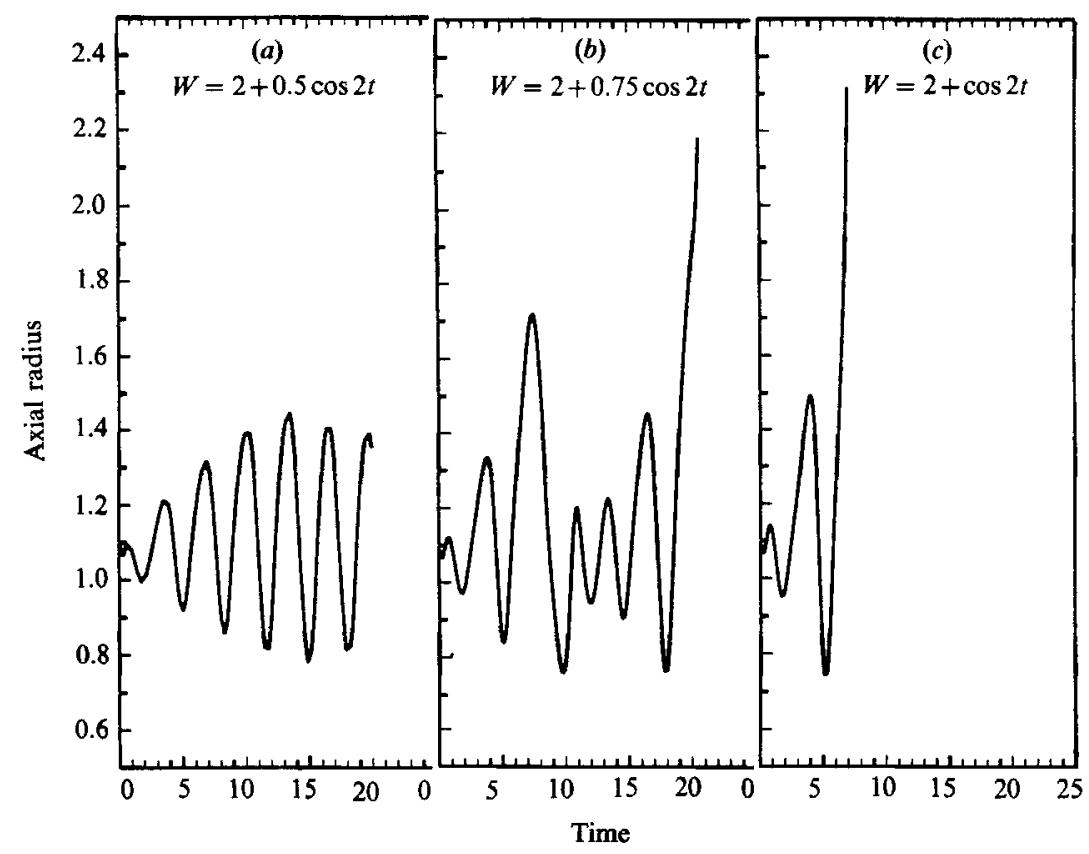

Fioure 11. Time-dependent numerical solutions of the full fluid mechanics problem for resonant forcing of different amplitudes (the case of the time-periodic variation of surface tension)

In (37), $\kappa_{(\phi)}$ is the normal curvature in the $\phi$-direction, $W$ is the (dimensionless) Weber number, and $\tau_{\xi \xi}$ is the total normal stress, which includes both static and dynamic pressure and viscous stress contributions. The far-field boundary condition for the uniaxial straining flow is given as

$$
\psi \sim \frac{1}{2} x \sigma^{2}, \quad \omega \rightarrow 0 \quad \text { as } \quad \xi \rightarrow 0 \quad\left(\left(x^{2}+\sigma^{2}\right)^{\frac{1}{2}} \rightarrow \infty\right) .
$$

The dimensionless parameters in (33) and (37) are defined as

$$
W(t)=\frac{2 \rho(E a)^{2} a}{\gamma(t)}, \quad R=\frac{2 \rho(E a) a}{\mu} .
$$

From the above equations, we can see that the effect of a time-period variation of surface tension is represented by $W(t)$ in the normal stress condition (37). In our numerical experiments for time-periodic variations of surface tension, the Weber number for the case of potential flow $(\mu=0)$ was varied as

$$
W(t)=W_{0}(1+\epsilon \cos \omega t),
$$

where $W_{0}=2, \omega=2$, and $\epsilon=0.25,0.375,0.5$. For the potential flow limit $(\mu=0)$, the unperturbed system has a critical Weber number of 2.76 , and the intrinsic frequency $\left(\omega_{0}\right)$ is 2 at $W=2$ (see Kang \& Leal 1987). The forcing frequency was chosen as $\omega=$ 2 to induce a resonance. As we can see in figure 11, three different types of bubble behaviour are observed as the amplitude of the time-periodic forcing increases. There is a regular (two-timescale) motion for $\epsilon=0.25$ (figure 11 $a$ ), a non-regular (possibly chaotic) motion that leads to breakup for $\epsilon=\mathbf{0 . 3 7 5}$ (figure $11 b$ ), and an early stage bubble breakup for $\epsilon=0.5$ (figure 11c). The three different behaviours may be explained in the context of the model analysis. The region of regular motion in the 


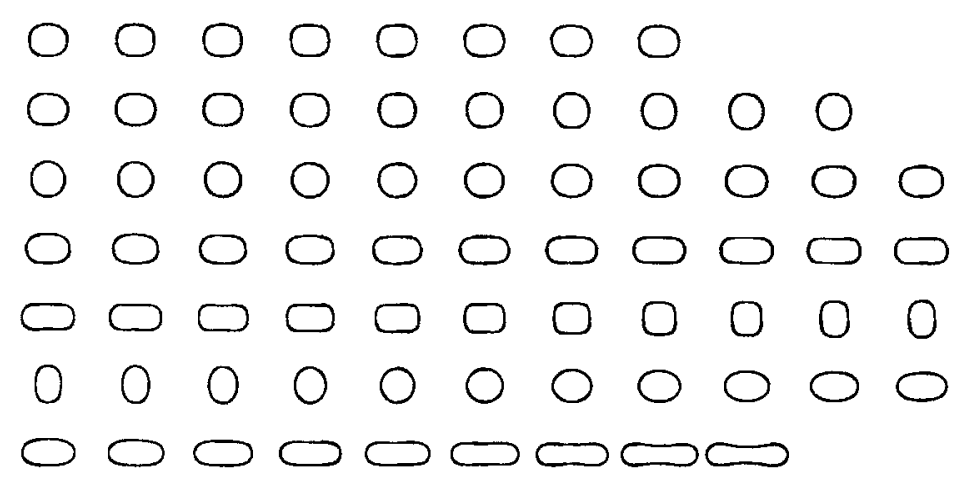

Figure 12. Consecutive bubble deformations for the case of $W=2+\cos 2 t$ in figure $11 \mathrm{c}$ ).

(Time interval between images, $\Delta t=0.1$.)

Poincaré map shrinks as the forcing amplitude increases (see figure 5). Therefore, some initial points can be in the regular region for smaller forcing, while the same points are in the chaotic region for larger forcing. It is also worth noting that the regular motion for $\epsilon=0.25$ can be best described by two different timescales as predicted by the two-timing method for the model analysis. In figure 12, consecutive bubble deformations are shown for the case of $W=2+\cos 2 t$. As we can see clearly, a resonant forcing with sufficient strength induces an early stage bubble breakup. A more detailed explanation of the dynamical results from the numerical experiment will be given in the next subsection for the more realistic case of a time-periodic strain rate.

$$
\text { 7.2. The case of } E(t)=E(t+T) \text { and } \dot{\gamma}=0
$$

Now, let us turn to the numerical results for our main topic of this paper, the effect of time-periodic straining flow on the bubble dynamics. For this case, we define $E_{0}$ as a representative (e.g. time-average) characteristic strain rate, and nondimensionalize using a the following characteristic scales:

$$
l_{\mathrm{e}}=a, \quad t_{\mathrm{c}}=E_{0}^{-1}, \quad U_{\mathrm{e}}=E_{0} a .
$$

If we define the Weber number and the Reynolds number as

$$
W_{0}=\frac{2 \rho\left(E_{0} a\right)^{2} a}{\gamma(t)}, \quad R=\frac{2 \rho\left(E_{0} a\right) a}{\mu},
$$

then the governing equations and boundary conditions are the same as the case in \$7.1, except for the normal stress condition

$$
\tau_{\xi \xi}-\frac{4}{W_{0}}\left(\kappa_{(\eta)}+\kappa_{(\phi)}\right)=0
$$

and the boundary condition at infinity

$$
\psi \sim \frac{1}{2}\left(\frac{W(t)}{W_{0}}\right)^{\frac{1}{2}} x \sigma^{2}, \quad \omega \rightarrow 0 \quad \text { as } \quad \xi \rightarrow 0 \quad\left(\left(x^{2}+\sigma^{2}\right)^{\frac{1}{2}} \rightarrow \infty\right) .
$$

In contrast to the previous section, the time variation of the Weber number appears in the far-field boundary condition rather than the normal stress condition. 


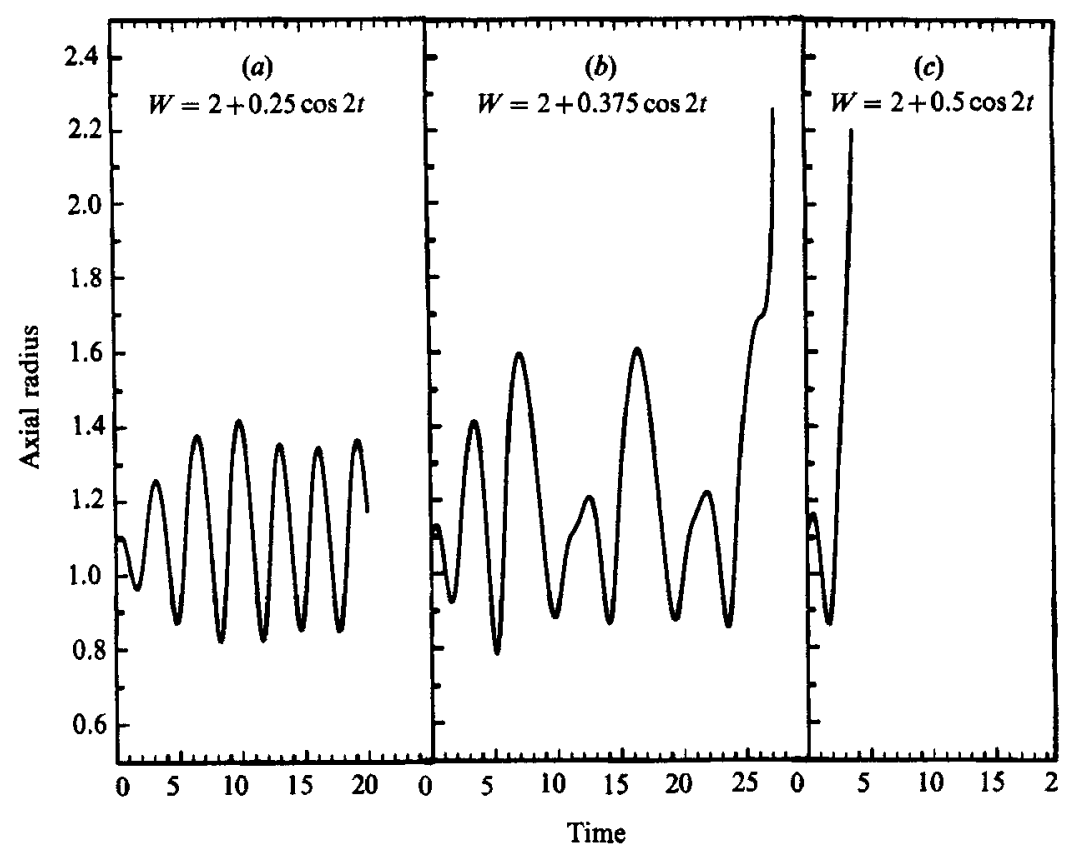

Figure 13. Time-dependent numerical solutions of the full fluid mechanics problem for resonant forcing of different amplitudes (the case of the time-periodic strain rate).

The results of numerical experiments for the potential flow case $(\mu=0)$ are presented in figure 13. The Weber numbers in the experiment were varied as

$$
W(t)=W_{0}(1+\epsilon \cos \omega t)
$$

where $W_{0}=2, \omega=2$, and $\epsilon=0.125,0.1875,0.25$. The same values for $W_{0}$ and $\omega$ are chosen as for the case of a time-periodic variation of surface tension, but smaller values of $\epsilon$ are used for this case. As we can see in figure 13, three quite distinctive bubble dynamics are observed as $\epsilon$ increases.

At $\epsilon=0.125$ (figure 13a), the bubble dynamics can be well described by two different timescales, i.e. a short-timescale oscillation is imbedded in the longtimescale variation. Especially, it is worth noting that the time intervals between extrema are quite even, and are very close to the period of forcing. As we increase $\epsilon$ to 0.1875 (figure $13 b$ ), the dynamical behaviour is quite different from the $\epsilon=0.125$ case. It seems quite irregular and the time intervals between the extrema are much larger than the forcing period. Further, after several irregular oscillations, the bubble is extended indefinitely. This behaviour can be best understood in terms of homoclinic tangling. As explained in $\$ 6.2$, the initial condition is in a certain lobe (a lobe is a region like $R$ in figure $7 a$ ) and it is mapped to the other lobes according to the lobe dynamics. Eventually the bubble state is pumped out to the exterior region (of breakup) by the map $R \rightarrow P(R)$ in our model analysis, after which it is extended indefinitely, which leads to bubble breakup. The fundamental difference between this case and the regular case for $\epsilon=\mathbf{0 . 1 2 5}$ is that the same initial condition is in the regular region for $\epsilon=0.125$, where the lobes formed by the homoclinic tangle do not penetrate (see figure $9 a$ ). Therefore if the initial condition is in the regular region, there is no mechanism to map the state to the breakup region. However, as we increase the forcing amplitude, the region covered by the lobes (i.e. the chaotic region 


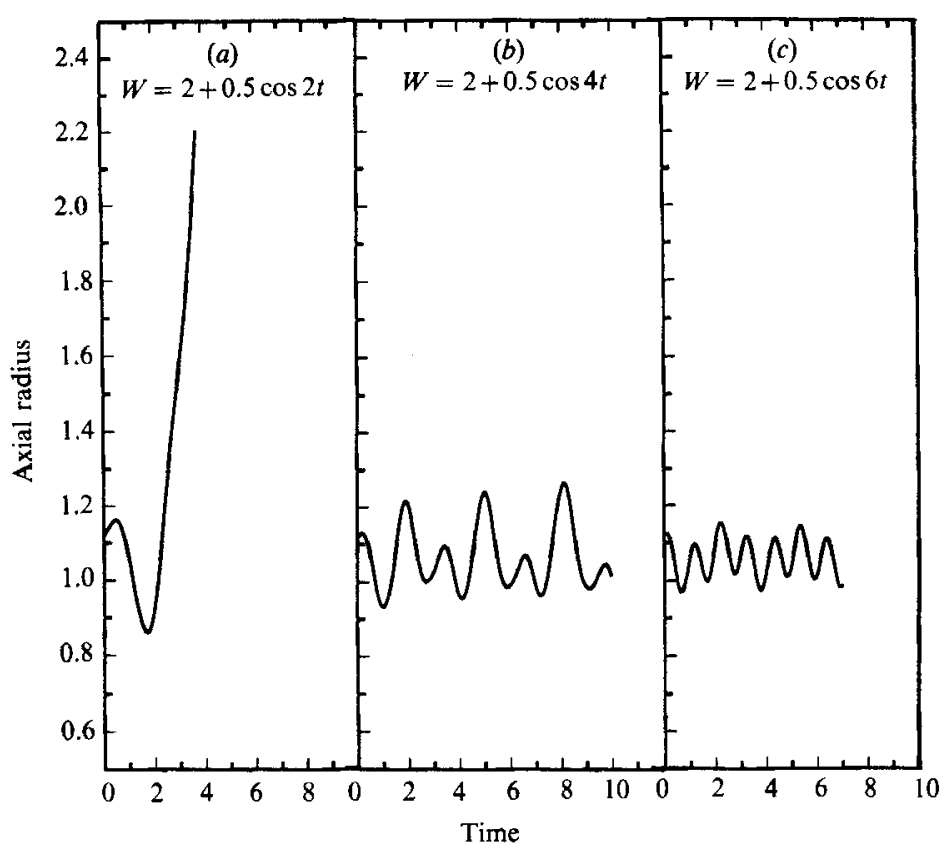

Figure 14. The effect of the forcing frequency on the bubble dynamics in a time-periodic strain flow (numerical solution for the full fluid mechanics problem).

in our model discussion) increases, and the same initial condition can be in the chaotic region for larger forcing amplitude. As we further increase the amplitude to $\epsilon=0.25$ (figure $13 c$ ), we observe an early stage breakup. This fact can be again well understood in terms of the lobe dynamics. The increase of the forcing amplitude results in thicker and larger lobes, as discussed in \$5.2, and this increases the likelihood of transfer from $R \rightarrow P(R)$ after a small number of periods.

From the results of the two sets of numerical experiments described above, we see that the detailed bubble dynamies in the time-periodic-straining case is of course different from the case of time-periodic variations of the surface tension. However, the qualitative features are the same for both cases, and well explained by the simple model analysis.

So far we have tested the effect of the forcing amplitude. In the next subsection, we test the effect of forcing frequency when the amplitude is fixed.

\subsection{The effect of the variation of the forcing frequency}

From the model analysis, we have seen that the effect of forcing frequency is dramatic and there is an optimal frequency for bubble breakup. In this subsection, we shall demonstrate the same effect via numerical results for the full fluid mechanics problem.

From $\$ 7.2$, we have seen that we can obtain an early stage breakup by using resonant forcing $W(t)=2(1+0.25 \cos 2 t)$, in spite of the fact that the average Weber number 2 is much lower than the critical Weber number 2.76 for the constant-strain case. Now, it is quite interesting to see what happens if we change the frequency while holding the amplitude fixed. In the following numerical experiment for a timeperiodic strain rate, we changed the Weber number according to

$$
W(t)=2(1+0.25 \cos \omega t)
$$


where $\omega=2,4$, and 6 . Since $\omega=2$ is the resonant frequency, $\omega=4$ and $\omega=6$ are two and three times of the resonant frequency. As we can see in figure 14, the effect of time-periodic forcing is reduced drastically as the frequency is increased. The output amplitude is much smaller than for the resonant forcing case (in fact, for the resonant case we obtained an early stage breakup), and the oscillatory behaviour is quite regular. In the case of $\omega=4=2 \omega_{0}$ (figure $14 b$ ), we can clearly see that the $\omega=4$ mode is superposed on the intrinsic $\omega=2$ mode (note that the interval between the maxima is very close to $\left.\pi=2 \pi / \omega_{0}\right)$. One more interesting fact is that the primary maxima and minima are slowly increasing while the two intermediate extrema are slowly decreasing. In other words, the difference between the primary maximum and intermediate maximum increases and the difference between the primary and intermediate minimum decreases. This may be explained by the second-order resonant effect as we have seen in \$6.1.2. The fixed point is a saddle point for the long-timescale dynamics for double-frequency forcing. Therefore, if the two maxima are near the unstable manifolds and the two minima are near the stable manifolds, the long-timescale dynamics will appear exactly as in the numerical results. The result for the $\omega=6=3 \omega_{0}$ (figure $14 c$ ) case also exhibits very regular behaviour corresponding to a superposition of the dynamics of the $\omega=2$ and $\omega=6$ modes. It should be noted that the amplitude is even smaller than the $\omega=4$ case.

\section{Discussion}

From the previous section, although the data presented are limited, we have seen that the dynamical features predicted by the simple dynamic model are very faithfully reproduced in the numerical experiments for the full fluid mechanics problem. This fact may seem surprising at first in the sense that the dynamics of a very complicated problem in fluid mechanics can be effectively understood by a simple nonlinear oscillator model. However, it is not a mystery at all. Although the detailed local dynamics of the problem is, of course, determined by the details of the specific problem, the global dynamical features are more or less determined by the presence of the homoclinic orbit in the dynamical solution for the constant-strainrate potential flow problem. In order to have a homoclinic orbit, there must be at least one centre and one saddle point in the dynamical solution for a given set of parameters, as shown by the stability results in $\S 3$. Furthermore, as we have discussed in $\$ 5$, we have a double-zero codimension-2 bifurcation point at the limit point for the potential flow problem. Then, by the argument in $\$ 5$, the global dynamical behaviour must be like our model at least near the critical point. The above argument may explain why the very simple model can predict the dynamical features of the extremely difficult and complicated free boundary problem in fluid mechanics.

As mentioned in $\$ 5$ for the unfolding theory, the dynamic model considered in the present paper is not limited to the problem of bubble dynamics in straining flows. In fact, the general form for the two-dimensional model has been derived using the unfolding theory based under the following general assumptions :

(i) The critical point is a limit point for the existence of steady-state solutions and its linear stability equation has double-zero eigenvalues.

(ii) For the non-dissipative case, the system behaviour can be effectively described by the Hamiltonian dynamics.

(iii) The ideal non-dissipative system (e.g. the potential flow problem) is the limit for $\mu \rightarrow 0$ of the real problem with non-zero viscosity (i.e. the potential flow problem 
is a uniformly valid first approximation - this is often true for fluid mechanics problems involving zero shear stress-free boundaries, but is not generally true for problems involving no-slip boundaries).

If the above three conditions are satisfied, the dynamics near the critical point for a time-periodic (or steady) forcing must be qualitatively similar to the dynamics predicted in the previous sections. This includes a number of important problems involving the motions of bubbles and drops. Among many examples, we briefly discuss the following two problems which show the same behaviour (see the references cited for detailed discussions): (i) growth of a spherical gas bubble in weakly viscous fluid; (ii) dynamics of an inviscid drop in a uniform electric field.

The exact dynamics of the first problem is described by the Rayleigh-Plesset equation, in which the measure of deformation is the radius and the driving force is the difference between the pressure inside the bubble and the pressure at infinity. The Rayleigh-Plesset equation has been studied by many investigators. Among them, recently Chang \& Chen (1986) applied a normal form bifurcation anaylsis near the critical point, and showed explicitly that the dynamics for a constant pressure difference near the critical point is described by the dynamical system (13) (or equivalently by (12) for the left half-plane of the two-dimensional parameter space). Furthermore, they concluded that there is no limit cycle for physically meaningful situations, based on an argument that is very similar to that presented here in $\S 5$. Therefore, if we allow a small time-periodic variation in the pressure driving force, we can expect precisely the same qualitative dynamics for the radius $R$ that are discussed in the present paper for the deformation variable $x$. An analysis of some aspects of the transition between regular and chaotic behaviour was published recently by Smereka, Birnir \& Banerjee (1987).

The second problem was originally considered by Taylor (1964) who showed that there is a limit point for the existence of steady-state solutions. To our knowledge, no detailed dynamical analysis is yet available for this problem. However, the dynamics near the critical point satisfies the three conditions given above, and the qualitative behaviour will again correspond to the results obtained here. The reason is that in the case of zero viscosity no mechanism plays a role to damp out oscillations of shape about the steady-state shape (i.e. eigenvalues of the linear stability problem are purely imaginary at subcritical parameter values). In addition, at the critical point (limit point), two eigenvalues (a pair of conjugate imaginary eigenvalues (from the $P_{2}(\cos \theta)$-mode become zero to satisfy the bifurcation condition. Thus, the dynamics near the critical point must be qualitatively the same as the dynamics of a bubble in straining flows.

So far, we have pointed out that a single, simple set of equations describes the qualitative dynamics of a drop (or a bubble) in many problems where the underlying physics is different for each specific problem. Finally, we want to stress that the dynamic analysis in this paper has significant implication for realistic applications. In such cases, the driving force for deformation is rarely maintained at a constant amplitude and frequency, but rather it is usually given by a very complicated time function. In the usual analysis of experimental results, reliance has been placed on the relevance of some sort of time-averaged magnitude for the force (strain rate, electric field strength, pressure difference, etc.). For example, existing criteria for bubble breakup in time-dependent flows are based upon the concept of a critical 'mean' value for the Weber number, with the critical value obtained from the corresponding steady problem. However, the present analysis implies that a simpleminded application of the steady analysis based on a time-averaged value for the 
forcing is not enough, and that the results from a dynamical analysis should also be considered. We are currently considering the generalization of the results in this paper to more general time histories, and to detailed analyses for the related physical problems that are described above.

This work was supported by a grant from the office of Naval Research.

\section{REFERENCES}

Chang, H.-C. \& Chen, L.-H. 1986 Phys. Fluids 29, 3530-3536.

Guck enheimer, J. \& Holmes, P. 1983 Nonlinear Oscillations, Dynamical Systems, and Bifurcations of Vector Fields. Springer.

Kang, I. S. \& Leal, L. G. 1987 Phys. Fluids 30, 1929-1940.

KANG, I. S. \& LEAL, L. G. 1988 J. Fluid Mech. 187, 231-266.

Kevorkiax, J. \& Cole, J. D. 1981 Perturbation Methods in Applied Mathematics. Springer.

LAмв, H. 1932 Hydrodynamics, 6th edn. Dover.

Miksis, M. 1981 Phys. Fluids 24, 1229-1231.

RoM-Kedar, V. 1988 Part I: An analytical study of transport, mixing and chaos in an unsteady vortical flow. Part II : Transport in two dimensional maps. Ph.D. dissertation, California Institute of Technology.

RYSkin, G. \& LEAL, L. G. 1984 J. Fluid Mech. 148, 37-43.

Smerkek, P., Birnir, B. \& Banerjez, S. 1987 Phys. Fluids 30, 3342.

TAkens, F. 1974 Publ. Math. IHES 43, 47-100.

TAYlOR, G. I. 1964 Proc. R. Soc. Lond. A 280, 383-397.

Tsamopoulos, J. A., Akylas, T. R. \& Brown, R. A. 1985 Proc. Roy. Soc. Lond. A 401, 67-88.

Wiggins, S. 1988 Global Bifurcations and Chaos-Analytical Methods. Springer. 